\title{
Estimates of population parameters and consumption/biomass ratio for fishes in reservoirs, Paraná State, Brazil
}

\author{
Éder André Gubiani ${ }^{1}$, Luiz Carlos Gomes ${ }^{2}$ and Angelo Antonio Agostinho²
}

\begin{abstract}
Consumption, natural mortality, and growth are important parameters for the construction for both fisheries and ecosystems models. We estimated k (growth constant of the von Bertalanffy's function), $\mathrm{L}_{o}$ (asymptotic length), W (asymptotic weight), Ar (caudal fin index), M (natural mortality), and Q/B (consumption/biomass ratio) for several fish species inhabiting reservoirs. We explored possible relationships among these parameters for 135 fish species sampled in thirty reservoirs in the State of Paraná, southern Brazil. Maximum length $\left(\mathrm{L}_{\max }\right)$ varied from 2.60 to $79.00 \mathrm{~cm}, \mathrm{~L}_{o}$ from 2.73 to $85.05 \mathrm{~cm}, \mathrm{~W}_{o}$ from 0.23 to $9,490.26 \mathrm{~g}, \mathrm{k}$ from 0.01 to $2.38 \mathrm{yr}^{-1}$, Ar from 0.03 to $3.60, \mathrm{M}$ from 0.16 to $2.82 \mathrm{yr}^{-1}$, and $\mathrm{Q} / \mathrm{B}$ from 3.31 to 67.18 . Significant correlations were observed between most pairs of parameters, except for $\mathrm{Ar}$ and $\mathrm{k}$, Ar and $\mathrm{L}_{o o}$, and for $\mathrm{M}$ and $\mathrm{Q} / \mathrm{B}$. The estimates presented here may be useful for constructing Ecopath models, both in Brazil and other tropical regions, where the use of ecosystem modeling is growing.

O consumo, a mortalidade natural e as estimativas de crescimento são parâmetros importantes para a construção de modelos pesqueiros e ecossistêmicos. Dessa forma, neste trabalho foram calculados os parâmetros k (constante de crescimento da equação de von Bertalanffy), $\mathrm{L}_{o o}$ (comprimento assintótico), $\mathrm{W}_{o o}$ (peso assintótico), Ar (índice da nadadeira caudal), $\mathrm{M}$ (mortalidade natural) e Q/B (consumo/biomassa) para peixes em reservatórios. Além disso, foram exploradas possíveis relações entre esses parâmetros para 135 espécies de peixes amostradas em 30 reservatórios no estado do Paraná, sul do Brasil. O comprimento máximo $\left(\mathrm{L}_{\max }\right.$ ) variou de 2,60 a 79,00 cm, $\mathrm{L}_{o o}$ de 2,73 a 85,05 cm, $\mathrm{W}_{o o}$ de 0,23 a 9.490,26 g, k de 0,01 a 2,38 ano ${ }^{-1}, \mathrm{Ar}$ de 0,03 a 3,60, M de 0,16 a 2,82 ano $^{-1}$, e Q/B de 3,31 a 67,18. Correlações significativas foram observadas entre todos os pares de parâmetros, exceto para Ar e k, Ar e $\mathrm{L}_{o o}$ e para $\mathrm{M}$ e Q/B. As estimativas aqui apresentadas podem ser úteis para a construção de modelos Ecopath, tanto em outras regiões tropicais, como no Brasil, onde os modelos ecossistêmicos estão em amplo estágio de desenvolvimento.
\end{abstract}

Key words: Ecosystem approach, Lentic ecosystems, Population's variables.

\section{Introduction}

Estimates of growth parameters for fish species are important not only to understand their life history and behavior, but also to manage fishery resources, since they allow estimating the potential productivity (Nikolsky, 1969; Gulland, 1977). Several studies point out to the lack of important biological parameters to estimate yield and give support to management, especially for tropical fish (Pauly, 1998a, 1998b; Beverton, 1998; Holt, 1998). Worldwide, it is known approximately 28,000 fish species (Nelson, 2006), among these only $1,400(5 \%)$ have information on growth available (Binohlan \& Pauly, 2000); in the Neotropical region such information should be even less available.
The knowledge of growth parameters (e.g. von Bertalanffy growth constant $\mathrm{k}$ and asymptotic length $\mathrm{L}_{o \text { o, }}$ maximum reported length and age $\left(\mathrm{L}_{\max }\right.$ and $\mathrm{t}_{\max }$, respectively) as well as the consumption/biomass ratio $(\mathrm{Q} / \mathrm{B})$, and natural mortality (M) are important parameters for building ecosystems models (e.g. Ecopath; Christensen \& Pauly, 1993), several other fishery models and, consequently, managing fishery resources (Froese \& Pauly, 2000; Froese \& Binohlan, 2000, 2003). In addition, these parameters allow testing life history theories and obtaining preliminary estimates of other parameters from those already available (Froese \& Binohlan, 2000, 2003; Stergiou \& Karpouzi, 2002; Stergiou \& Karachle, 2006).

Examples of use of empirical equations for parameters estimation described above include asymptotic length from

\footnotetext{
${ }^{1}$ Universidade Estadual do Oeste do Paraná, Centro de Engenharias e Ciências Exatas, Gerpel, Grupo de Estudos em Recursos Pesqueiros e Limnologia, Programa de Pós-Graduação em Recursos Pesqueiros e Engenharia de Pesca. Rua da Faculdade, 645, 85903-000 Toledo, PR, Brazil.egubiani@yahoo.com.br(EAG)

${ }^{2}$ Universidade Estadual de Maringá, Programa de Pós-Graduação em Ecologia de Ambientes Aquáticos Continentais, Departamento de Biologia, Nupélia. Av. Colombo, 5790, 87020-000 Maringá, PR, Brazil.1cgomes@nupelia.uem.br(LCG), agostinhoaa@nupelia.uem.br (AAA)
} 
maximum reported length, natural mortality from growth parameters, optimum exploitation length from asymptotic length or length at first maturity, length at first maturity from asymptotic length and/or von Bertallanfy growth constant, trophic level from maximum reported length (Pauly, 1980; Froese \& Binohlan, 2000; Stergiou \& Karpouzi, 2002; Angelini \& Agostinho, 2005a).

Recently, there has been an increase in the number of studies using the ecosystem approach, which justifies compiling the cited parameters values for future use in the building of Ecopath models. Moreover, information on Neotropical fishes can be uploaded to online databases, such as the FishBase (Froese \& Pauly, 2010). Thereby, in this study, we estimated/compiled the parameters k (growth constant of the VBGF), $\mathrm{L}_{o o}$ (asymptotic length), $\mathrm{L}_{\text {max }}$ (maximum reported length), $\mathrm{W}_{o o}$ (asymptotic weight), Ar (caudal fin index), M (natural mortality) and Q/B (consumption/biomass) for 135 Neotropical fish species collected in 30 reservoirs in the State of Paraná, southern Brazil. In addition, we evaluated possible significant relationships between $\mathrm{k}$ and $\mathrm{L}_{\max }, \mathrm{k}$ and $\mathrm{L}_{o o} \mathrm{k}$ and $\mathrm{W}_{o o}, \mathrm{k}$ and $\mathrm{M}, \mathrm{k}$ and $\mathrm{Ar}, \mathrm{k}$ and $\mathrm{Q} / \mathrm{B}, \mathrm{L}_{o o}$ and $\mathrm{Q} / \mathrm{B}, \mathrm{W}_{o o}$ and $\mathrm{L}_{\max }$, $\mathrm{M}$ and $\mathrm{Ar}$, and $\mathrm{M}$ and $\mathrm{L}_{\max }$. Finally, we made a trophic categorization for all species considered in this study. All analyses were performed aiming at detecting patterns in the life history of those fish species.

\section{Material and Methods}

Study area. The study reservoirs were located in six river basins (Paranapanema, Tibagi, Ivaí, Piquiri, Iguaçu, and Leste) throughout the State of Paraná and its borders with the States of São Paulo and Santa Catarina (Fig. 1; Table 1). Although the main purpose of these reservoirs is energy production, they are also used for water supply, and for fishing and leisure. Reservoirs were selected to include a diversity of areas, morphometries, hydraulic retention times and distribution throughout the state. More detailed description of some characteristics of each reservoir is presented in Table 1.

Sampling. Fishes were sampled in 30 studied reservoirs (Table 1); two samplings were carried out, one in July and another in November 2001. Samplings were conducted in the lacustrine zone of the reservoirs (following Thornton et al., 1990) with 20-m-long gillnets of diverse mesh sizes $(2.4,3,4,5,6,7,8,9$, 10,12 , and $14 \mathrm{~cm}$ opposite knots), set for $24 \mathrm{~h}$ and checked every $8 \mathrm{~h}$. Seine nets ( $20 \mathrm{~m}$ long and $0.8 \mathrm{~cm}$ mesh) were used during the day in the littoral.

All fishes caught were fixed in formalin (4\%), stored in polyethylene containers identified with date and capture site, and then sent to the laboratory of Núcleo de Pesquisas em Limnologia, Ictiologia e Aquicultura (Nupélia; Universidade Estadual de Maringá- UEM) for further analysis. Fish were identified according to Severi \& Cordeiro (1994), Ingenito et al. (2004), Oyakawa et al. (2006), Graça \& Pavanelli (2007) and Menezes et al. (2007), measured for length (total and standard, $\mathrm{cm}$ ), and weight (total body weight, $\mathrm{g}$ ).
Data analyses. Trophic categorization of most species was based on studies conducted in the reservoirs evaluated here. However, when it was not possible we used information from the literature. For this, additional references included Fugi \& Hahn (1991), Hahn et al. (1991, 1992, 1997a, 1997b, 1998, 1999), Andrian et al. (1994), Souza-Stevaux et al. (1994), Andrian \& Barbieri (1996), Ferreti et al. (1996), Lolis \& Andrian (1996), Loureiro \& Hahn (1996), Agostinho et al. (1997), Almeida et al. (1997), Gealh \& Hahn (1998), Abujanra et al. (1999), Fugi et al. (2001, 2005), Ortêncio Filho et al. (2001), Cassemiro et al. (2002, 2003, 2005), Abelha \& Goulart (2004), Russo et al. (2004), Abelha et al. (2005, 2006), Bennemann et al. (2005, 2006), Loureiro-Crippa \& Hahn (2006), Oricolli \& Bennemann (2006), and Cantanhêde et al. (2008).

For species with more than 50 individuals the parameters $\mathrm{k}$ and $\mathrm{L}_{o o}$ were estimated from the sampled data. Growth parameters were obtained by fitting von Bertalanffy's growth constant function (VBGF; Ricker, 1975), which calculates the parameters $\mathrm{k}$ (growth constant) and $\mathrm{L}_{o}$ (asymptotic length), as follows:

$$
\mathrm{L}_{\mathrm{t}}=\mathrm{L}_{\infty} \times\left(1-\mathrm{e}^{-\mathrm{k}\left(\mathrm{t}-\mathrm{t}_{0}\right)}\right),
$$

where $\mathrm{L}_{\mathrm{t}}$ is the total fish length $(\mathrm{cm})$ at age $\mathrm{t}, \mathrm{L}_{o}$ is the asymptotic length $(\mathrm{cm}), \mathrm{k}$ is VBGF's inclination or growth rate $\left(\mathrm{year}^{-1}\right)$, and $\mathrm{t}_{0}$ is the age intercept (fish age is assumed to be zero).

Asymptotic length $\left(\mathrm{L}_{o o}\right)$ and k were calculated, whenever possible, using length-frequency data and FISAT's ELEFAN I routine (Sparre et al., 1989; Gayanilo \& Pauly, 1997), with the option of scanning for k-values. This routine estimates VBGF's adherence to length frequency data. Sometimes, the bestfitted $k$ value was corroborated by the literature or by FISAT's routine "by eye", which fits the VBGF visually. When the collected data did not allow fitting, we used published data (Perez Lizama \& Vazzoler, 1993; Angelini \& Agostinho, 2005a; Froese \& Pauly, 2010; Table 2). When it was not possible to obtain VBGF parameters, $\mathrm{L}_{o o}$ was estimated using the length of the largest individual of the population sample: $\mathrm{L}_{o o}=\mathrm{L}_{\max }$ * 1.05 (Angelini \& Agostinho, 2005a).

Production, according to Ivlev (1945), is the "total quantity of tissue elaborated by a fish population during a stated period of time, although not all individuals survive until the end of that period". As the production/biomass $(\mathrm{P} / \mathrm{B})$ ratio is hard to estimate directly, it can be considered equivalent to total mortality $[Z=$ fishing mortality $(F)+$ natural mortality $(\mathrm{M})]$ (Pauly et al., 2000). The study reservoirs have negligible fishing activity, if any. Thus, as shown by Allen (1971) under equilibrium conditions, the $\mathrm{P} / \mathrm{B}$ ratio is similar to natural mortality $(\mathrm{M})$, which was calculated with the empiric regression of Pauly (1980):

$$
\mathrm{M}=\mathrm{k}^{0.65} \times \mathrm{L}_{\infty}^{-0.279} \times \mathrm{T}^{0.463}
$$

where $\mathrm{M}$ is natural mortality (year ${ }^{-1}$ ), $\mathrm{k}$ and $\mathrm{L}_{o}$ are the VBGF's parameters, and $\mathrm{T}$ is the average annual water temperature (average for the 30 reservoirs; in ${ }^{\circ} \mathrm{C}$ ).

There are several empirical approaches to estimate consumption/biomass ratio $(\mathrm{Q} / \mathrm{B})$, and this parameter is 


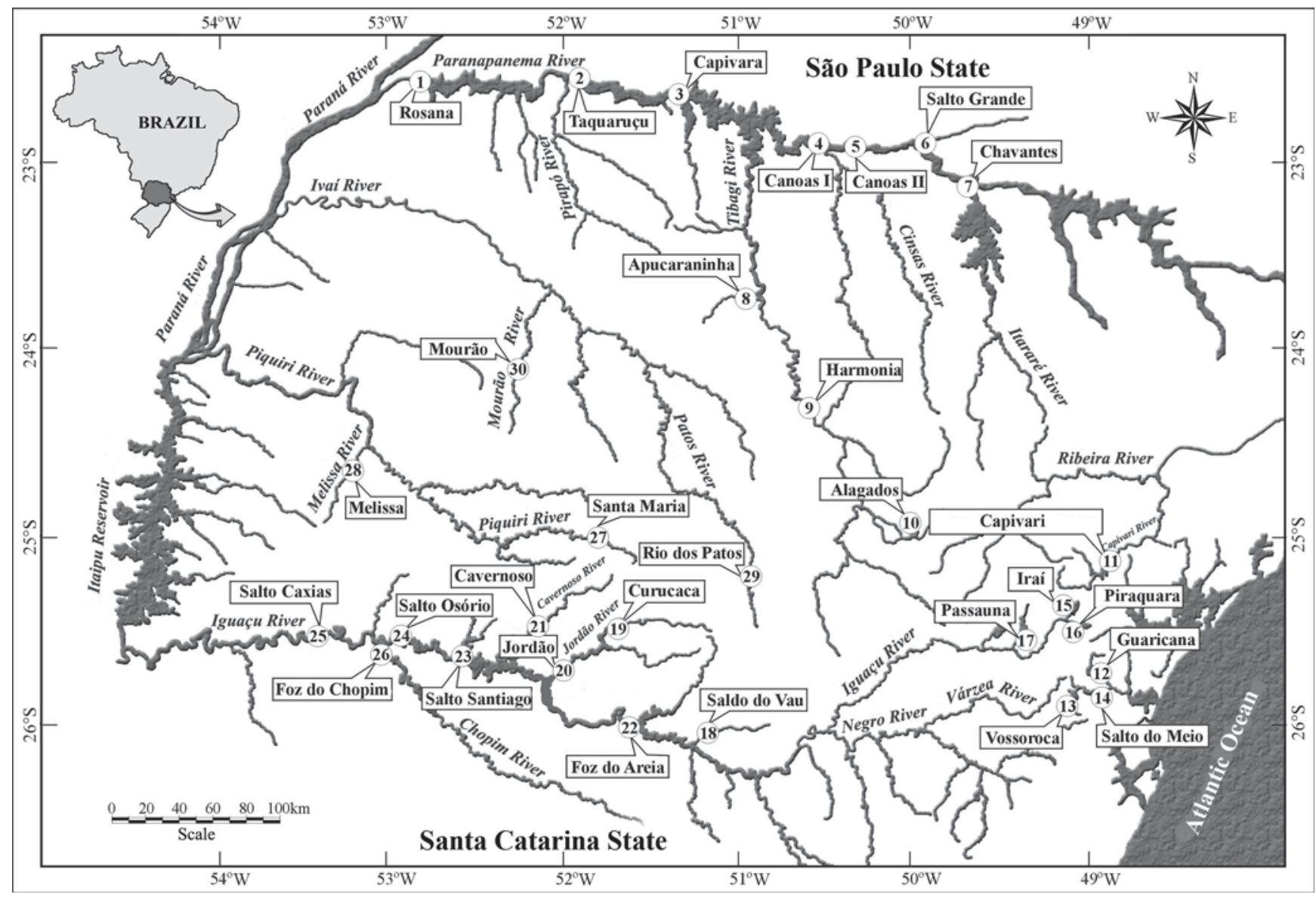

Fig. 1. Location of the 30 reservoirs in the State of Paraná sampled in July and November 2001.

relatively easy to estimate for fish populations (Palomares \& Pauly, 1989, 1998; Pauly, 1989; Pauly et al., 1990; Jarre et al., 1991). It is interesting to notice that $Q / B$ values for the same species vary as a result of using different empirical formulas and predictor parameters; for example, there might be variation in measurements of proportion and asymptotic weight, as well as in the type of food consumed by a species. To estimate $\mathrm{Q} / \mathrm{B}$ we used the empiric regression model proposed by Palomares \& Pauly (1998), as follows:

$\log \mathrm{Q} / \mathrm{B}=7.964-0.204 \log \mathrm{W}_{\infty}-1.965 \mathrm{~T}^{\prime}+0.083 \mathrm{Ar}+0.532 \mathrm{H}+0.398 \mathrm{D}$ where $\mathrm{Q} / \mathrm{B}$ is consumption/biomass rate $\left(\right.$ year $\left.^{-1}\right), \mathrm{W}_{o}$ is the asymptotic weight (wet weight in $\mathrm{g}$, estimated based on the length/weight relationship for $\mathrm{L}_{o o}$ ); $\mathrm{T}^{\prime}$ is the inverse of the average annual water temperature $\left(T^{\prime}=1000 / G+273.15\right)$, where $G$ is the average water temperature of the 30 reservoirs $\left({ }^{\circ} \mathrm{C}\right)$; Ar is the caudal fin index $\left(\mathrm{Ar}=\mathrm{h}^{2} / \mathrm{s}\right.$, where $\mathrm{h}$ is the caudal fin height ( $\mathrm{mm}$ ) and $\mathrm{s}$ is the caudal fin surface area $\left(\mathrm{mm}^{2}\right)$, extending to the narrowest part of the caudal peduncle) and a measure of the swimming and metabolic activity of the fish (Palomares \& Pauly, 1998). Ar values were estimated by measurements of five individuals, when possible, per species (analyzed individuals were deposited in the collection of Museu de Ictiologia, Nupélia). Variables $\mathrm{H}$ and $\mathrm{D}$ refers to feeding behavior, i.e., $\mathrm{H}=1$ for herbivorous, $\mathrm{D}=1$ for detritivorous and $\mathrm{H}=\mathrm{D}=0$ for carnivorous. Values of average annual water temperature, for both equations listed above, were provided by the limnology laboratory of Nupélia (Table 1).

We tested significant relationships between $\mathrm{k}$ and $\mathrm{L}_{\text {max }}$, $\mathrm{k}$ and $\mathrm{L}_{o}, \mathrm{k}$ and $\mathrm{W}_{o o}, \mathrm{k}$ and $\mathrm{M}, \mathrm{k}$ and $\mathrm{Ar}, \mathrm{k}$ and $\mathrm{Q} / \mathrm{B}, \mathrm{L}_{o}$ and $\mathrm{Q} /$ $\mathrm{B}, \mathrm{W}_{o o}$ and $\mathrm{L}_{\max }, \mathrm{M}$ and $\mathrm{Ar}$, and $\mathrm{M}$ and $\mathrm{L}_{\max }$ with linear regressions. All these relationships are relevant to the study of life history (Jensen, 1997; Froese \& Pauly, 2000; Froese \& Binohlan, 2000, 2003).

\section{Results}

$\mathrm{L}_{\max }$ varied from 2.60 to $79.00 \mathrm{~cm}$. $\mathrm{L}_{o o}$ from 2.73 to 85.05 $\mathrm{cm}$. The values of $\mathrm{L}_{o}$ for 34 species (Table 2) were compiled from the literature; among these species $C$. modestus, $A$. altiparanae, P. britskii, A. gymnogenys, G. brasiliensis, $H$. aff. malabaricus, $R$. quelen and $S$. melanodermatum showed $\mathrm{L}_{o o}$ less than the $\mathrm{L}_{\max }$ recorded in this study. $\mathrm{W}_{o}$ from 0.23 to $9,490.26 \mathrm{~g}$, $\mathrm{k}$ from 0.01 to $2.38 \mathrm{yr}^{-1}$, Ar from 0.03 to $3.60, \mathrm{M}$ from 0.16 to $2.72 \mathrm{yr}^{-1}$, and $\mathrm{Q} / \mathrm{B}$ from 3.31 to 67.18 (Table 2).

The 135 fish species captured were categorized in six trophic categories (detritivorous, herbivorous, insectivorous, 
Table 1. Some characteristics of the study reservoirs ( ${ }^{\mathrm{a}}$ - Perimeter estimated in Google ${ }^{\mathrm{TM}}$ Earth. Depth $=$ maximum depth, R.T. $=$ Hydraulic retention time, A.A.W.T. = average annual water temperature). Consulted references: Júlio Jr. et al. (2005), Angelini \& Gomes (2008), Companhia Paranaense de Energia Elétrica (2007), Comitê Brasileiro de Barragens (2007).

\begin{tabular}{|c|c|c|c|c|c|c|c|c|c|c|}
\hline Reservoir & River & Basin & $\begin{array}{c}\text { Damming } \\
\text { Year }\end{array}$ & Basin area & Area $\left(\mathrm{km}^{2}\right)$ & Perimeter $^{\mathrm{a}}(\mathrm{km})$ & Depth (m) & R.T. (days) & Volume $\left(\mathrm{km}^{3}\right)$ & $\begin{array}{c}\text { A.A.W.T. } \\
\left({ }^{\circ} \mathrm{C}\right)\end{array}$ \\
\hline$\overline{\text { Taquaruçu }}$ & Paranapanema & Paranapanema & 1992 & 88000 & 80.1 & $156.35^{\mathrm{a}}$ & 26.5 & 7.9 & 672.5 & 23.6 \\
\hline Capivara & Paranapanema & Paranapanema & 1975 & 85000 & 419.3 & $738.93^{\mathrm{a}}$ & 52.5 & 126.8 & 10540.0 & 23.7 \\
\hline Canoas II & Paranapanema & Paranapanema & 1999 & 39556 & 22.5 & $65.81^{\mathrm{a}}$ & 16.5 & 4.4 & 140.0 & 23.5 \\
\hline Canoas I & Paranapanema & Paranapanema & 1999 & 40920 & 30.8 & $74.64^{\mathrm{a}}$ & 26.0 & 6.0 & 207.0 & 23.9 \\
\hline Salto Grande & Paranapanema & Paranapanema & 1958 & 38600 & 12.0 & $24.91^{\mathrm{a}}$ & 9.2 & 1.4 & 44.2 & 21.9 \\
\hline Chavantes & Paranapanema & Paranapanema & 1970 & 27500 & 400.0 & $401.84^{\mathrm{a}}$ & 78.0 & 352.7 & 8795.0 & 21.9 \\
\hline Rosana & Paranapanema & Paranapanema & 1986 & 99000 & 220.0 & $230.32^{\mathrm{a}}$ & 26.0 & 18.6 & 1920.0 & 24.1 \\
\hline Alagados & Pitangui & Tibagi & 1945 & 377 & 7.2 & 47.20 & 9.2 & 46.0 & 0.03 & 19.8 \\
\hline Apucaraninha & Apucaraninha & Tibagi & 1958 & 513 & 2.0 & 16.90 & - & 12.0 & 0.015 & 20.3 \\
\hline Harmonia & Harmonia & Tibagi & 1950 & - & 3.0 & $7.49^{\mathrm{a}}$ & 12.0 & - & 8.0 & 20.9 \\
\hline Patos & Patos & Ivaí & 1949 & 1086 & 1.3 & 3.20 & 5.7 & 0.2 & 0.0008 & 18.5 \\
\hline Mourão & Mourão & Ivaí & 1964 & 573 & 11.3 & 60.00 & 12.7 & 70.0 & 0.065 & 20.9 \\
\hline Cavernoso & Cavernoso & Iguaçu & 1965 & 1460 & 2.9 & $3.73^{\mathrm{a}}$ & 8.3 & - & - & 19.5 \\
\hline Salto Santiago & Iguaçu & Iguaçu & 1979 & 43900 & 208.0 & $376.99^{\mathrm{a}}$ & 78.0 & - & 6753.0 & 21.6 \\
\hline Salto Osório & Iguaçu & Iguaçu & 1975 & 45800 & 51.0 & $180.16^{\mathrm{a}}$ & 43.0 & - & 1270.0 & 21.2 \\
\hline Salto Caxias & Iguaçu & Iguaçu & 1998 & 57000 & 124.0 & $267.14^{\mathrm{a}}$ & 53.0 & - & 3573.0 & 21.8 \\
\hline Foz do Chopim & Chopim & Iguaçu & 1970 & 3800 & 2.9 & $5.25^{\mathrm{a}}$ & 6.0 & - & - & 20.1 \\
\hline Jordão & Jordão & Iguaçu & 1996 & 4700 & 3.4 & $21.74^{\mathrm{a}}$ & 60.0 & - & 110.0 & 18.4 \\
\hline Curucaca & Jordão & Iguaçu & 1982 & - & 2.0 & $3.70^{\mathrm{a}}$ & 10.5 & - & - & 17.5 \\
\hline Foz do Areia & Iguaçu & Iguaçu & 1980 & 29900 & 139.0 & $288.67^{\mathrm{a}}$ & 135.0 & - & 5779.0 & 20.7 \\
\hline Passauna & Passaúna & Iguaçu & 1978 & - & 14.0 & $35.30^{\mathrm{a}}$ & - & 420.0 & - & 16.5 \\
\hline Piraquara & Piraquara & Iguaçu & 1979 & - & 3.3 & 40.00 & 18.0 & 438.0 & 0.5 & 19.9 \\
\hline Salto do Vau & Palmital & Iguaçu & 1959 & 340 & 2.0 & - & 3.5 & - & 0.0003 & 16.2 \\
\hline Iraí & Iraí & Iguaçu & 2000 & - & 15.0 & $20.12^{\mathrm{a}}$ & 8.5 & 240.0 & - & 20.0 \\
\hline Santa Maria & Piquiri & Piquiri & 1950 & - & 0.1 & - & 4.3 & - & - & 16.7 \\
\hline Melissa & Melissa & Piquiri & 1962 & 368 & 2.9 & - & 5.3 & - & - & 17.9 \\
\hline Guaricana & Arraial & Leste & 1957 & - & 7.0 & 7.20 & 17.0 & 13.0 & 0.00683 & 19.9 \\
\hline Vossoroca & São João & Leste & 1949 & 160 & 5.1 & $18.49^{\mathrm{a}}$ & 12.5 & - & 0.04 & 19.0 \\
\hline Salto do meio & Cubatão & Leste & 1931 & 252 & 0.1 & $3.90^{\mathrm{a}}$ & 6.2 & - & 0.0004 & 18.3 \\
\hline Capivari & Capivari & Leste & 1970 & - & 12.0 & 123.50 & 43.0 & 48.0 & 156.0 & 19.9 \\
\hline
\end{tabular}

invertivorous, omnivorous, and piscivorous; Table 2). Insectivorous (30 species), detritivorous (29 species) and piscivorous (26 species) were the trophic categories with higher number of species, respectively. Omnivorous $(23$ species), invertivorous (15 species) and herbivorous (12 species) showed smaller number of species (Table 2).

Various relationships were apparent with the VBGF. In this sense, $\mathrm{k}$ was significant negatively related to maximum length, and asymptotic length and weight $(\mathrm{F}=45.00, \mathrm{~F}=62.80$ and $\mathrm{F}=59.60$, respectively; Figs. 2a, b, c). The piscivorous species $R$. quelen showed low annual growth rate $(\mathrm{k}=0.01$ year $^{-1}$; Table 2) and low natural mortality, whereas $O$. niloticus $\left(\mathrm{k}=2.38\right.$ year $^{-1}$; Table 2$)$ showed the opposite trend; both were considered outliers (Fig. 2). On the other hand, relationships between $\mathrm{k}$ and caudal fin index, and natural mortality ( $\mathrm{F}=458.38$; Fig. 2e) and consumption/biomass ratio $(\mathrm{F}=30.43$; Fig. $2 \mathrm{f}$ ) were positive.

The relationship between $\mathrm{k}$ and caudal fin index was not significant $(\mathrm{F}=0.02$; Fig. 2d). The fin shape of knifefishes $G$. sylvius and E. trilineata strongly affected the relationship (Fig. 2d); but even after their exclusion the relationship was still non-significant $(\mathrm{F}=0.16 ; \mathrm{p}=0.68)$. Rhamdia quelen and $O$. niloticus did not follow the general relationship between $\mathrm{k}$ and consumption/biomass ratio (Fig. 2f).

The relationship between asymptotic length and consumption/biomass ratio was negative $(\mathrm{F}=78.33$; Fig. 3a).
Interestingly, the species above the regression line in Figure 3 a represent small- and medium-sized species that are mostly detritivorous and have high population growth rates. The relationship between maximum length and asymptotic weight was positive ( $\mathrm{F}=563.14$; Fig. $3 \mathrm{~b}$ ). The species $M$. salmoides (located below the regression line) and L. macrocephalus (located above the regression line) were outliers.

The relationship between natural mortality and maximum length was negative ( $\mathrm{F}=136.37$; Fig. $3 \mathrm{c}$ ). The species $L$. macrocephalus was an outlier; when this fish was removed, the relationship showed stronger non-linear tendency (Spearman $\rho=-0.80 ; p<0.05$ ). The relationship between natural mortality and caudal fin index was not significant $(\mathrm{F}=0.09$; Fig. 3d), following the same trend observed for the relationship between $\mathrm{k}$ and caudal fin index. Similarly, after exclusion of the knifefishes, the relationship was still non-significant $(\mathrm{F}=$ $0.06 ; \mathrm{p}=0.80)$.

\section{Discussion}

In this study we obtained significant relationships among various biological parameters. These relationships can be useful to obtain other estimates, such as yield per recruit (Y/R), which can be easily calculated from $\mathrm{L}_{\max }, \mathrm{W}_{\max }, \mathrm{t}_{\max }$, and Ar, among others. Significant negative relationships were established between the parameters $\mathrm{k}$ and $\mathrm{L}_{\max }, \mathrm{k}$ and $\mathrm{L}_{o \infty}$ and $\mathrm{k}$ and $\mathrm{W}_{\infty}$. On 
Table 2. Demographic and biological attributes of 135 fish species captured in 30 reservoirs in the State of Paraná, southern Brazil, in July and November 2001. $\mathrm{N}=$ number of individuals captured, $\mathrm{TC}=$ species' trophic category, $\mathrm{L}_{\max }=$ maximum length $(\mathrm{cm}), \mathrm{L}_{o o}=$ asymptotic length ( $\mathrm{cm}$; values with asterisk were obtained of the literature), $\mathrm{W}_{o}=$ asymptotic weight $(\mathrm{g}), \mathrm{k}=\mathrm{growth}$ constant from von Bertalanffy's equation $\left(\right.$ year $\left.^{-1}\right), \mathrm{Ar}=$ caudal fin index (Palomares \& Pauly, 1998), M = natural mortality $\left(\right.$ year $\left.^{-1}\right)$ and $\mathrm{Q} / \mathrm{B}=$ consumption/biomass ratio $\left(\right.$ year $\left.^{-1}\right) .{ }^{a}$ - Froese \& Pauly $(2010),{ }^{\mathrm{b}}$ - Angelini \& Agostinho (2005a), ${ }^{\mathrm{c}}-\mathrm{Perez}$ Lizama \& Vazzoler (1993). *For species in which k was calculate, Rn (goodness of fit index) was greater than 0.6.

\begin{tabular}{|c|c|c|c|c|c|c|c|c|c|}
\hline Species & $\mathrm{N}$ & $\mathrm{TC}$ & $\mathrm{L}_{\max }$ & $\mathrm{L}_{\infty}$ & $\mathrm{W}_{\infty}$ & $\mathrm{k}^{*}$ & $\mathrm{Ar}$ & $\mathrm{M}$ & $\mathrm{Q} / \mathrm{B}$ \\
\hline Apareiodon affinis (Steindachner, 1879) & 1096 & detritivorous & 15.70 & 16.48 & 55.35 & 0.50 & 1.26 & 1.17 & 30.54 \\
\hline Apareiodon ibitiensis Campos, 1944 & 3 & detritivorous & 4.80 & 5.00 & 1.42 & $0.50^{\mathrm{a}}$ & 1.17 & 1.63 & 63.37 \\
\hline Astyanax minor Garavello \& Sampaio, 2010 & 5564 & detritivorous & 13.50 & $13.65^{*}$ & 156.18 & 0.50 & 2.18 & 1.08 & 29.46 \\
\hline Apareiodon piracicabae (Eigenmann, 1907 & 39 & detritivorous & 11.00 & 11.55 & 18.37 & $0.50^{\mathrm{a}}$ & 1.27 & 1.29 & 38.29 \\
\hline Apareiodon vittatus Garavello, 1977 & 170 & detritivorous & 14.60 & $17.64 *$ & 56.60 & 0.95 & 1.37 & 1.73 & 31.04 \\
\hline Astyanax jordanensis Alcaraz, Pavanelli \& Bertaco, 2009 & 1 & detritivorous & 15.40 & 9.90 & 9.95 & $0.50^{\mathrm{a}}$ & 1.20 & 1.35 & 42.85 \\
\hline Cyphocharax modestus (Fernández-Yépez, 1948) & 115 & detritivorous & 22.00 & $18.00^{*}$ & 180.52 & $0.80^{\mathrm{b}}$ & 0.81 & 1.80 & 27.72 \\
\hline Deuterodon sp. B (sensu C.S Pavanelli) & 945 & detritivorous & 11.70 & 12.28 & 26.13 & 0.80 & 0.94 & 1.72 & 33.45 \\
\hline Deuterodon sp. D (sensu C.S Pavanelli) & 1985 & detritivorous & 12.80 & 13.44 & 33.88 & 0.80 & 1.17 & 1.68 & 33.19 \\
\hline Hypostomus ancistroides (Ihering, 1911) & 27 & detritivorous & 23.30 & 24.46 & 161.07 & $0.45^{\mathrm{a}}$ & 0.52 & 0.98 & 21.33 \\
\hline Hypostomus commersoni Valenciennes, 1836 & 68 & detritivorous & 38.20 & $44.62 *$ & 2270.64 & 0.24 & 2.09 & 0.55 & 16.77 \\
\hline Hypostomus derbyi (Hanseman, 1911) & 110 & detritivorous & 40.00 & 42.00 & 638.00 & 0.51 & 2.09 & 0.92 & 21.73 \\
\hline Hypostomus hermanni (Ihering, 1905) & 1 & detritivorous & 11.50 & 12.10 & 27.86 & $0.50^{\mathrm{a}}$ & 0.71 & 1.27 & 31.65 \\
\hline Hypostomus margaritifer (Regan, 1908) & 1 & detritivorous & 16.20 & 17.00 & 58.66 & $0.30^{\mathrm{a}}$ & 0.75 & 0.83 & 27.37 \\
\hline Hypostomus myersi (Gosline, 1947) & 34 & detritivorous & 22.50 & $25.20 *$ & 188.05 & 0.49 & 1.77 & 1.02 & 26.23 \\
\hline Hypostomus nigromaculatus (Schubart, 1964) & 1 & detritivorous & 11.50 & 12.10 & 23.08 & $0.50^{\mathrm{a}}$ & 0.57 & 1.27 & 31.98 \\
\hline Hypostomus regani (Ihering, 1905) & 23 & detritivorous & 24.30 & 25.50 & 215.49 & $0.25^{\mathrm{a}}$ & 0.46 & 0.66 & 19.87 \\
\hline Hypostomus topavae (Godoy, 1969) & 51 & detritivorous & 20.00 & 21.00 & 142.00 & 0.50 & 0.94 & 1.09 & 23.70 \\
\hline Hypostomus multidens Jerep, Shibatta \& Zawadzki, 2007 & 10 & detritivorous & 29.60 & 31.08 & 385.25 & 0.50 & 0.94 & 0.98 & 19.33 \\
\hline Hypostomus iheringii (Regan, 1908) & 5 & detritivorous & 29.60 & $36.33 *$ & 468.03 & $0.20^{\mathrm{a}}$ & 0.35 & 0.52 & 16.60 \\
\hline Hypostomus strigaticeps (Regan, 1908) & 1 & detritivorous & 20.40 & 21.40 & 146.84 & $0.50^{\mathrm{a}}$ & 0.71 & 1.09 & 22.52 \\
\hline Proloricaria prolixa (Isbrücker \& Nijssen, 1978) & 63 & detritivorous & 38.60 & 40.53 & 359.87 & $0.20^{\mathrm{a}}$ & 0.41 & 0.50 & 17.72 \\
\hline Loricaria sp. & 15 & detritivorous & 27.70 & 29.10 & 110.00 & 0.55 & 1.16 & 1.06 & 26.04 \\
\hline Loricariichthys platymetopon Isbrücker \& Nijssen, 1979 & 10 & detritivorous & 31.40 & 33.00 & 254.44 & $0.55^{\mathrm{b}}$ & 1.16 & 1.10 & 21.95 \\
\hline Megalancistrus parananus (Peters, 1881) & 4 & detritivorous & 35.50 & 37.30 & 865.82 & $0.26^{\mathrm{b}}$ & 1.56 & 0.60 & 18.47 \\
\hline Prochilodus lineatus (Valenciennes, 1836) & 61 & detritivorous & 63.70 & $85.05^{*}$ & 9490.26 & 0.34 & 2.16 & 0.60 & 12.70 \\
\hline Rhinelepis aspera Spix \& Agassiz, 1829 & 3 & detritivorous & 34.50 & 36.20 & 825.70 & $0.39^{\mathrm{b}}$ & 0.76 & 0.80 & 16.00 \\
\hline Rineloricaria sp. & 71 & detritivorous & 16.30 & $20.58 *$ & 52.56 & 0.43 & 0.45 & 0.99 & 26.41 \\
\hline Steindachnerina brevipinna (Eigenmann \& Eigenmann, 1889) & 42 & detritivorous & 16.80 & 17.64 & 85.60 & $0.86^{\mathrm{b}}$ & 1.39 & 1.63 & 28.62 \\
\hline Astyanax bifasciatus Garavello \& Sampaio, 2010 & 7351 & herbivorous & 19.00 & $19.16^{*}$ & 69.74 & 1.00 & 1.40 & 1.81 & 40.73 \\
\hline Astyanax dissimilis Garavello \& Sampaio, 2010 & 8 & herbivorous & 13.80 & $16.28^{*}$ & 57.40 & $0.44^{\mathrm{a}}$ & 1.50 & 1.00 & 43.20 \\
\hline Astyanax longirhinus Garavello \& Sampaio, 2010 & 1163 & herbivorous & 8.40 & 8.80 & 9.60 & 0.80 & 1.05 & 1.89 & 57.07 \\
\hline Bryconamericus iheringii (Boulenger, 1887) & 905 & herbivorous & 8.00 & 8.40 & 7.68 & $0.70^{\mathrm{a}}$ & 1.66 & 1.75 & 67.18 \\
\hline Ctenopharyngodon idella (Valenciennes, 1844) & 1 & herbivorous & 43.20 & 45.40 & 965.06 & $0.15^{\mathrm{a}}$ & 1.56 & 0.40 & 24.59 \\
\hline Hypophthalmichthys molitrix (Valenciennes, 1844) & 1 & herbivorous & 40.00 & 42.00 & 1151.20 & $0.16^{\mathrm{a}}$ & 0.93 & 0.48 & 31.78 \\
\hline Hypophthalmus edentatus Spix \& Agassiz, 1829 & 4 & herbivorous & 47.80 & 50.20 & 1874.82 & $0.28^{\mathrm{a}}$ & 2.04 & 1.60 & 27.48 \\
\hline Leporinus lacustris Campos, 1945 & 1 & herbivorous & 15.50 & 16.30 & 91.00 & $0.80^{\mathrm{a}}$ & 1.15 & 1.59 & 36.79 \\
\hline Metynnis lippincottianus (Cope, 1870) & 24 & herbivorous & 14.70 & 15.43 & 92.64 & $0.70^{\mathrm{a}}$ & 1.26 & 1.48 & 37.45 \\
\hline Schizodon borellii (Boulenger, 1900) & 8 & herbivorous & 28.00 & 29.40 & 516.00 & $0.42^{\mathrm{b}}$ & 2.03 & 1.40 & 30.54 \\
\hline Schizodon nasutus Kner, 1858 & 234 & herbivorous & 33.70 & 35.38 & 567.49 & $0.34^{\mathrm{a}}$ & 1.41 & 0.73 & 26.62 \\
\hline Tilapia rendalli (Boulenger, 1897) & 228 & herbivorous & 31.50 & $39.27 *$ & 3064.45 & 0.31 & 1.48 & 0.42 & 19.12 \\
\hline Aphyocharax anisitsi Eigenmann \& Kennedy, 1903 & 2 & insectivorous & 4.50 & 4.70 & 1.30 & $0.90^{\mathrm{a}}$ & 0.80 & 2.43 & 24.06 \\
\hline Aphyocharax dentatus Eigenmann \& Kennedy, 1903 & 6 & insectivorous & 7.50 & 7.90 & 5.00 & $0.80^{\mathrm{a}}$ & 1.37 & 1.95 & 20.36 \\
\hline Apteronotus sp. & 1 & insectivorous & 30.00 & $30.20 *$ & 224.00 & $0.60^{\mathrm{a}}$ & 0.53 & 1.11 & 7.99 \\
\hline Astyanax altiparanae Garutti \& Britski, 2000 & 3550 & insectivorous & 15.90 & $14.70^{*}$ & 80.87 & 0.79 & 1.24 & 1.35 & 12.34 \\
\hline Astyanax fasciatus (Cuvier, 1819) & 50 & insectivorous & 16.00 & 16.80 & 96.29 & $0.25^{\mathrm{c}}$ & 0.86 & 0.74 & 10.69 \\
\hline
\end{tabular}

the other hand, $\mathrm{k}$ and $\mathrm{M}$ and $\mathrm{k}$ and $\mathrm{Q} / \mathrm{B}$ ratio showed significant positive relationships. The first relationship can be used for estimating $\mathrm{k}$ from the corresponding $\mathrm{L}_{\max }$ values, which are easier to be obtained. Stergiou \& Karachle (2006) also found significant negative relationship between $\mathrm{k}$ and $\mathrm{L}_{\max }$ for 142 fish stocks from the Greek Seas. Perez Lizama \& Vazzoler (1993), in a review on marine and freshwater fish growth in Brazil, observed the same trend in the relationship between $\mathrm{L}_{o}$ and $\mathrm{k}$. The negative correlation between $\mathrm{k}$ and $\mathrm{L}_{o o}$ and $\mathrm{k}$ and $\mathrm{W}_{\infty}$ seems to be a recurrent pattern in fishes (Beverton \& Holt, 1959; Pauly, 1998a, 1998b; Froese \& Pauly, 2000).
The relationship between $\mathrm{k}$ and $\mathrm{L}$ should provide the slope value equal to -0.33 , when assuming the isometric growth (Jensen, 1997). Stergiou \& Karachle (2006) updated the slope value for all Greek fish stocks and found the value of -0.3006 , close to the theoretical -0.33 . We found a slope value of -0.6195 , which differed greatly from the theoretical value, as well as a correlation coefficient $(\mathrm{r}=-0.57)$, which is higher than verified by Stergiou \& Karachle (2006) for Greek Seas fish stocks $(\mathrm{r}=-0.34)$. In addition, if we remove $R$. quelen and $O$. niloticus (which deviated from the regression line) from the analysis, the value of the slope becomes even higher $(-0.8211 ; \mathrm{r}=-0.64)$. 
Table 2 (cont.). Demographic and biological attributes of 135 fish species captured in 30 reservoirs in the State of Paraná, southern Brazil, in July and November 2001. $\mathrm{N}$ = number of individuals captured, $\mathrm{TC}=$ species' trophic category, $\mathrm{L}_{\max }=$ maximum length $(\mathrm{cm}), \mathrm{L}_{o}=$ asymptotic length ( $\mathrm{cm}$; values with asterisk were obtained of the literature), $\mathrm{W}_{o o}=$ asymptotic weight $(\mathrm{g}), \mathrm{k}=$ growth constant from von Bertalanffy's equation (year $\left.{ }^{-1}\right), \mathrm{Ar}=$ caudal fin index (Palomares \& Pauly, 1998), $\mathrm{M}=$ natural mortality $\left(\right.$ year $\left.^{-1}\right)$ and $\mathrm{Q} / \mathrm{B}=$ consumption/biomass ratio $\left(\right.$ year $\left.^{-1}\right){ }^{a}{ }^{a}$ - Froese \& Pauly (2010), ${ }^{b}$ - Angelini \& Agostinho (2005a), ${ }^{\mathrm{c}}$ - Perez Lizama \& Vazzoler (1993). *For species in which $\mathrm{k}$ was calculate, $\mathrm{Rn}$ (goodness of fit index) was greater than 0.6.

\begin{tabular}{|c|c|c|c|c|c|c|c|c|c|}
\hline Species & $\mathrm{N}$ & $\mathrm{TC}$ & $\mathrm{L}_{\max }$ & $\mathrm{L}_{\infty}$ & $\mathrm{W}_{\infty}$ & $\mathrm{k}^{*}$ & $\mathrm{Ar}$ & $\mathrm{M}$ & $\mathrm{Q} / \mathrm{B}$ \\
\hline Astyanax janeiroensis Eigenmann, 1908 & 129 & insectivorous & 14.00 & 14.70 & 46.62 & $0.80^{\mathrm{a}}$ & 1.19 & 1.64 & 12.47 \\
\hline Auchenipterus osteomystax (Ribeiro, 1918) & 286 & insectivorous & 26.50 & 27.82 & 194.51 & 0.50 & 1.47 & 1.01 & 9.84 \\
\hline Bryconamericus ikaa Casciotta, Almirón \& Azpelicueta, 2004 & 105 & insectivorous & 8.20 & 8.61 & 8.56 & 0.60 & 0.76 & 1.64 & 16.24 \\
\hline Bryconamericus sp. C (sensu C.S Pavanelli) & 94 & insectivorous & 7.30 & 7.66 & 5.84 & 0.70 & 1.19 & 1.80 & 19.07 \\
\hline Australoheros kaaygua Casciotta, Almirón \& Gómez, 2006 & 7 & insectivorous & 9.40 & $10.70^{*}$ & 30.60 & $0.40^{\mathrm{a}}$ & 0.73 & 1.14 & 12.46 \\
\hline Corydoras ehrhardti Steindachner, 1910 & 7 & insectivorous & 6.50 & 6.80 & 6.00 & $0.30^{\mathrm{a}}$ & 0.53 & 1.07 & 16.70 \\
\hline Corydoras carlae Nijssen \& Isbrücker, 1983 & 71 & insectivorous & 6.70 & 7.03 & 7.14 & 0.34 & 0.53 & 1.15 & 16.12 \\
\hline Crenicichla britskii Kullander, 1982 & 16 & insectivorous & 13.50 & 14.17 & 32.43 & $0.70^{\mathrm{a}}$ & 0.57 & 1.52 & 11.94 \\
\hline Crenicichla haroldoi Luengo \& Britski, 1974 & 70 & insectivorous & 19.90 & 20.89 & 97.49 & $0.60^{\mathrm{a}}$ & 0.60 & 1.23 & 9.60 \\
\hline Crenicichla niederleinii (Holmberg, 1891) & 53 & insectivorous & 22.80 & 23.94 & 164.51 & $0.65^{\mathrm{a}}$ & 0.62 & 1.25 & 8.65 \\
\hline Deuterodon sp. A (sensu C.S Pavanelli) & 782 & insectivorous & 13.70 & 14.38 & 51.01 & 0.75 & 1.10 & 1.58 & 12.05 \\
\hline Gymnotus inaequilabiatus (Valenciennes, 1836) & 8 & insectivorous & 37.50 & $63.00^{*}$ & 842.11 & $0.31^{\mathrm{c}}$ & 1.50 & 0.50 & 7.34 \\
\hline Hyphessobrycon eques (Steindachner, 1882) & 1 & insectivorous & 3.10 & 3.30 & 0.49 & $0.80^{\mathrm{a}}$ & 1.02 & 2.48 & 30.60 \\
\hline Aristichthys nobilis (Richardson, 1845) & 3 & insectivorous & 25.70 & 27.00 & 151.20 & $0.16^{\mathrm{a}}$ & 0.75 & 0.48 & 9.02 \\
\hline Leporellus vittatus (Valenciennes, 1850) & 11 & insectivorous & 20.00 & 21.00 & 98.06 & $0.34^{\mathrm{a}}$ & 0.66 & 0.85 & 9.69 \\
\hline Moenkhausia aff. intermedia Eigenmann, 1908 & 166 & insectivorous & 10.20 & 10.71 & 21.23 & $0.41^{\mathrm{b}}$ & 0.73 & 1.30 & 13.42 \\
\hline Pimelodella gracilis (Valenciennes, 1836) & 31 & insectivorous & 21.40 & 22.47 & 82.25 & $0.60^{\mathrm{a}}$ & 0.59 & 1.21 & 9.91 \\
\hline Pimelodus britskii Garavello \& Shibatta, 2007 & 979 & insectivorous & 37.60 & $35.49 *$ & 696.57 & 0.18 & 1.05 & 0.49 & 7.56 \\
\hline Rhamphichthys hahni (Meiken, 1937) & 1 & insectivorous & 51.00 & 53.60 & 224.00 & $0.50^{\mathrm{a}}$ & 0.50 & 1.01 & 7.94 \\
\hline Roeboides descalvadensis Fowler, 1932 & 13 & insectivorous & 8.00 & 8.40 & 5.66 & $0.85^{\mathrm{a}}$ & 1.40 & 1.99 & 19.99 \\
\hline Sternopygus macrurus (Bloch \& Schneider, 1801) & 1 & insectivorous & 34.50 & 36.20 & 67.57 & $0.50^{\mathrm{a}}$ & 0.50 & 0.94 & 10.14 \\
\hline Triportheus nematurus (Kner, 1858) & 5 & insectivorous & 24.00 & 25.20 & 187.00 & $0.70^{\mathrm{a}}$ & 1.09 & 1.29 & 9.22 \\
\hline Bryconamericus pyahu Azpelicueta, Casciotta \& Almirón, 2004 & 1 & insectivorous & 2.60 & 2.70 & 0.23 & $0.80^{\mathrm{a}}$ & 1.24 & 2.62 & 37.25 \\
\hline Bryconamericus sp. D (sensu C.S Pavanelli) & 4 & insectivorous & 7.10 & 7.50 & 5.00 & 0.70 & 1.54 & 1.81 & 21.03 \\
\hline Mimagoniates microlepis (Steindachner, 1876) & 42 & insectivorous & 5.30 & 5.56 & 1.91 & $0.75^{\mathrm{a}}$ & 0.61 & 2.06 & 21.45 \\
\hline Astyanax gymnogenys Eigenmann, 1911 & 1 & invertivorous & 15.60 & 16.40 & 5.82 & $0.74^{\mathrm{a}}$ & 0.74 & 1.90 & 17.52 \\
\hline Callichthys callichthys (Linnaeus, 1758) & 1 & invertivorous & 13.30 & 14.00 & 50.39 & $0.70^{\mathrm{a}}$ & 0.52 & 1.52 & 10.81 \\
\hline Characidium sp. & 1 & invertivorous & 5.20 & 5.50 & 1.45 & $0.80^{\mathrm{a}}$ & 0.67 & 2.15 & 22.95 \\
\hline Corydoras cf. paleatus (Jenyns, 1842) & 504 & invertivorous & 8.40 & $8.93 *$ & 12.93 & 0.74 & 1.29 & 1.78 & 16.52 \\
\hline Eigenmannia trilineata Lopes \& Castello, 1966 & 3 & invertivorous & 35.00 & 36.80 & 103.75 & $0.50^{\mathrm{a}}$ & 0.06 & 0.93 & 8.54 \\
\hline Geophagus brasiliensis (Quoy \& Gaimard, 1824) & 979 & invertivorous & 27.90 & $23.84^{*}$ & 507.05 & 0.37 & 1.20 & 1.54 & 8.53 \\
\hline Hemigrammus marginatus Ellis, 1911 & 3 & invertivorous & 3.80 & 4.00 & 0.73 & $0.80^{\mathrm{a}}$ & 0.78 & 2.35 & 26.92 \\
\hline Hoplosternum litoralle (Hancock, 1828) & 1 & invertivorous & 22.00 & 23.10 & 273.27 & $0.52^{\mathrm{b}}$ & 1.68 & 1.20 & 9.55 \\
\hline Iheringichthys labrosus (Lütken, 1874) & 571 & invertivorous & 33.50 & 35.17 & 439.55 & $0.55^{\mathrm{b}}$ & 1.63 & 1.10 & 8.59 \\
\hline Leporinus amblyrhynchus Garavello \& Britski, 1987 & 18 & invertivorous & 19.00 & 19.95 & 96.58 & $0.50^{\mathrm{a}}$ & 0.83 & 1.11 & 10.04 \\
\hline Odontesthes bonariensis (Valenciennes, 1835) & 53 & invertivorous & 41.80 & $52.50 *$ & 4138.51 & 0.24 & 0.85 & 0.48 & 4.68 \\
\hline Rhinodoras dorbignyi (Kner, 1855) & 2 & invertivorous & 19.00 & 20.00 & 157.82 & $0.30^{\mathrm{a}}$ & 0.86 & 0.79 & 9.13 \\
\hline Satanoperca pappaterra (Heckel, 1840) & 7 & invertivorous & 20.00 & 21.00 & 190.99 & $0.75^{\mathrm{a}}$ & 0.56 & 1.42 & 8.30 \\
\hline Gymnotus sylvius Albert \& Fernandes-Matioli, 1999 & 2 & invertivorous & 26.60 & 27.90 & 90.18 & $0.70^{\mathrm{a}}$ & 0.03 & 1.25 & 8.74 \\
\hline Odontostilbe sp. & 1 & invertivorous & 5.80 & 6.10 & 2.78 & $0.80^{\mathrm{a}}$ & 1.29 & 2.09 & 22.61 \\
\hline Astyanax aff. paranae Eigenmann, 1914 & 1334 & omnivorous & 15.60 & 16.38 & 70.42 & $0.75^{\mathrm{a}}$ & 1.80 & 1.52 & 12.89 \\
\hline Astyanax serratus Garavello \& Sampaio, 2010 & 8 & omnivorous & 14.20 & 14.90 & 50.26 & $0.70^{\mathrm{a}}$ & 1.10 & 1.49 & 12.07 \\
\hline Astyanax gymnodontus (Eigenmann, 1911) & 59 & omnivorous & 14.00 & 14.70 & 56.83 & $0.70^{\mathrm{a}}$ & 1.07 & 1.50 & 11.71 \\
\hline Bryconamericus stramineus Eigenmann, 1908 & 620 & omnivorous & 8.40 & 8.82 & 6.25 & $0.80^{\mathrm{a}}$ & 1.45 & 1.89 & 19.96 \\
\hline Colossoma macropomum (Cuvier, 1818) & 18 & omnivorous & 40.50 & 42.50 & 1246.46 & $0.23^{\mathrm{a}}$ & 3.60 & 0.54 & 9.93 \\
\hline
\end{tabular}

The parameters estimated here are consistent with other studies, which found that smaller species have higher $\mathrm{k}$ values (Pauly, 1998a, 1998b; Angelini \& Agostinho, 2005a). According to Pauly (1998a), the same asymptotic size may be associated with various $\mathrm{k}$ values. In addition, the same author showed that tropical fishes tend to be confined within a space defined by the smaller asymptotic size and higher $\mathrm{k}$ values than fish from temperate regions. Angelini \& Agostinho (2005a) estimated the $\mathrm{k}$ values for 35 fish species from the upper Paraná River floodplain and Itaipu reservoir and verified that fishes with smaller asymptotic lengths showed higher $\mathrm{k}$ values. In this study, $55 \%$ of species showed k value greater than 0.50; among these, except $H$. derbyi and P. mesopotamicus, all are considered small and medium-sized species. In addition, in reservoirs, the fish assemblage is composed basically by small-sized fishes (Dias et al., 2005; Agostinho et al., 2007). Those findings intensified the pattern highlighted by Pauly (1998a) for tropical fish. The large number of small and medium-sized species with fast growth is associated with the higher temperatures recorded in the tropics. Pauly (1998a) affirm that metabolic rates increase with temperature and that growth is limited by respiratory metabolism. Thus, tropical fishes should generally remain small. 
Table 2 (cont.). Demographic and biological attributes of 135 fish species captured in 30 reservoirs in the State of Paraná,

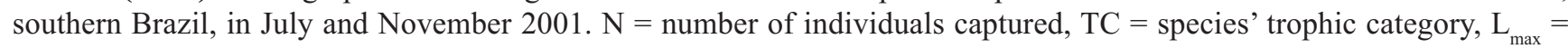
maximum length $(\mathrm{cm}), \mathrm{L}_{o o}=$ asymptotic length (cm; values with asterisk were obtained of the literature), $\mathrm{W}_{o o}=$ asymptotic weight $(\mathrm{g}), \mathrm{k}=$ growth constant from von Bertalanffy's equation (year $\left.{ }^{-1}\right), \mathrm{Ar}=$ caudal fin index (Palomares \& Pauly, 1998), M= natural mortality $\left(\right.$ year $\left.^{-1}\right)$ and $\mathrm{Q} / \mathrm{B}=$ consumption/biomass ratio $\left(\right.$ year $\left.^{-1}\right){ }^{\text {a }}$ - Froese \& Pauly (2010), ${ }^{\text {b }}$ - Angelini \& Agostinho (2005a), ${ }^{c}$ - Perez Lizama \& Vazzoler (1993). *For species in which k was calculate, Rn (goodness of fit index) was greater than 0.6.

\begin{tabular}{|c|c|c|c|c|c|c|c|c|c|}
\hline Species & $\mathrm{N}$ & $\mathrm{TC}$ & $\mathrm{L}_{\max }$ & $\mathrm{L}_{\infty}$ & $\mathrm{W}_{\infty}$ & $\mathrm{k}^{*}$ & $\mathrm{Ar}$ & M & $\mathrm{Q} / \mathrm{B}$ \\
\hline Cyprinus carpio Linnaeus, 1758 & 43 & omnivorous & 79.00 & $79.28^{*}$ & 8254.38 & 0.14 & 1.60 & 0.34 & 4.69 \\
\hline Deuterodon iguape Eigenmann, 1907 & 1060 & omnivorous & 14.50 & 15.22 & 50.23 & $0.80^{\mathrm{a}}$ & 0.80 & 1.62 & 11.41 \\
\hline Deuterodon sp. C & 12 & omnivorous & 9.70 & 10.20 & 14.18 & $0.70^{\mathrm{a}}$ & 0.95 & 1.66 & 15.20 \\
\hline Glanidium ribeiroi Hanseman, 1911 & 113 & omnivorous & 23.80 & $25.73^{*}$ & 465.88 & 0.22 & 1.73 & 0.60 & 8.65 \\
\hline Leporinus friderici (Bloch, 1794) & 11 & omnivorous & 36.60 & 38.40 & 1091.00 & $0.40^{\mathrm{b}}$ & 1.37 & 0.80 & 6.79 \\
\hline Leporinus macrocephalus Garavello \& Britski, 1988 & 1 & omnivorous & 10.1 & $42.00 *$ & 1983.96 & $0.07^{\mathrm{a}}$ & 1.13 & 0.24 & 5.74 \\
\hline Leporinus obtusidens (Valenciennes, 1836) & 28 & omnivorous & 37.10 & 38.95 & 1033.20 & $0.26^{\mathrm{b}}$ & 1.37 & 0.55 & 6.97 \\
\hline Leporinus octofasciatus Steindachner, 1915 & 21 & omnivorous & 23.80 & 24.99 & 226.20 & $0.22^{\mathrm{c}}$ & 1.25 & 0.61 & 9.14 \\
\hline Oreochromis niloticus (Linnaeus, 1758) & 3 & omnivorous & 22.00 & $30.00 *$ & 907.28 & $2.38^{\mathrm{a}}$ & 1.28 & 2.72 & 6.93 \\
\hline Parauchenipterus galeatus (Linnaeus, 1766) & 67 & omnivorous & 21.00 & 22.05 & 259.23 & $0.50^{\mathrm{b}}$ & 1.44 & 1.15 & 9.22 \\
\hline Phalloceros harpagos Lucinda, 2008 & 153 & omnivorous & 3.80 & 3.99 & 0.82 & $0.90^{\mathrm{a}}$ & 0.58 & 2.54 & 25.31 \\
\hline Piaractus mesopotamicus (Holmberg, 1887) & 49 & omnivorous & 53.00 & 55.65 & 3165.65 & $0.60^{\mathrm{a}}$ & 2.46 & 0.94 & 6.87 \\
\hline Pimelodus microstoma Steindachner, 1877 & 1 & omnivorous & 28.76 & 30.20 & 223.76 & $0.60^{\mathrm{a}}$ & 0.72 & 1.11 & 8.28 \\
\hline Pimelodus maculatus La Cépède, 1803 & 264 & omnivorous & 40.60 & 42.63 & 1050.45 & $0.43^{\mathrm{b}}$ & 1.64 & 0.90 & 7.20 \\
\hline Pimelodus ornatus Kner, 1858 & 2 & omnivorous & 32.50 & 34.10 & 542.24 & $0.30^{\mathrm{a}}$ & 0.56 & 0.68 & 6.71 \\
\hline Pimelodus ortmanni Hanseman, 1911 & 193 & omnivorous & 39.50 & 41.50 & 652.79 & 0.44 & 1.60 & 0.85 & 9.00 \\
\hline Pterodoras granulosus (Valenciennes, 1821) & 12 & omnivorous & 54.80 & 57.50 & 2006.55 & $0.35^{\mathrm{b}}$ & 1.35 & 0.70 & 6.03 \\
\hline Astyanax bockmanni Vari \& Castro, 2007 & 769 & omnivorous & 11.10 & $12.30 *$ & 32.00 & $0.44^{\mathrm{a}}$ & 2.30 & 1.20 & 16.66 \\
\hline Acestrorhynchus lacustris (Lütken, 1875) & 126 & piscivorous & 28.50 & 29.92 & 281.88 & $0.40^{\mathrm{b}}$ & 2.73 & 0.90 & 11.65 \\
\hline Ageneiosus militaris Valenciennes, 1836 & 14 & piscivorous & 24.19 & 25.40 & 133.32 & 0.35 & 2.49 & 0.80 & 12.91 \\
\hline Astronotus crassipinnis (Heckel, 1840) & 1 & piscivorous & 22.00 & 23.10 & 352.22 & $0.50^{\mathrm{a}}$ & 0.94 & 1.06 & 7.87 \\
\hline Cichla kelberi Kullander \& Ferreira, 2006 & 2 & piscivorous & 23.00 & 24.20 & 203.63 & $0.30^{\mathrm{a}}$ & 0.78 & 0.75 & 8.54 \\
\hline Clarias gariepinus (Burchell, 1822) & 1 & piscivorous & 64.70 & 67.90 & 2706.27 & $0.19^{\mathrm{a}}$ & 0.62 & 0.42 & 4.89 \\
\hline Crenicichla iguassuensis Hanseman, 1911 & 75 & piscivorous & 30.40 & $38.54 *$ & 759.27 & 0.10 & 0.98 & 0.32 & 6.78 \\
\hline Crenicichla yaha Casciotta, Almirón \& Gómez, 2006 & 47 & piscivorous & 30.60 & $36.75^{*}$ & 688.94 & 0.14 & 1.39 & 0.41 & 7.48 \\
\hline Galeocharax knerii (Steindachner, 1879) & 55 & piscivorous & 28.00 & 29.40 & 275.24 & $0.70^{\mathrm{a}}$ & 1.12 & 1.24 & 8.62 \\
\hline Hoplias aff. malabaricus (Bloch, 1794) & 433 & piscivorous & 56.80 & $53.13 *$ & 2514.86 & 0.42 & 1.30 & 0.90 & 5.70 \\
\hline Hoplias aff. lacerdae Ribeiro, 1908 & 1 & piscivorous & 51.30 & 53.90 & 1607.45 & $0.08^{\mathrm{a}}$ & 0.84 & 0.25 & 5.67 \\
\hline Ictalurus punctatus (Rafinesque, 1818) & 4 & piscivorous & 51.30 & $138.60 *$ & 20367.36 & $0.06^{\mathrm{a}}$ & 0.74 & 0.16 & 3.31 \\
\hline Micropterus salmoides (Lacépède, 1802) & 14 & piscivorous & 42.70 & 44.84 & 1730.40 & $0.14^{\mathrm{a}}$ & 0.63 & 0.69 & 21.50 \\
\hline Oligosarcus longirostris Menezes \& Géry, 1983 & 1768 & piscivorous & 30.40 & $30.98 *$ & 379.94 & 0.72 & 1.50 & 1.22 & 8.71 \\
\hline Oligosarcus paranensis Menezes \& Géry, 1983 & 1359 & piscivorous & 28.30 & $31.19 *$ & 341.48 & 0.70 & 2.02 & 1.22 & 9.74 \\
\hline Pinirampus pirinampu (Spix \& Agassiz, 1829) & 25 & piscivorous & 59.40 & 62.37 & 2368.59 & $0.22^{\mathrm{b}}$ & 2.48 & 0.50 & 7.19 \\
\hline Plagioscion squamosissimus (Heckel, 1840) & 294 & piscivorous & 46.90 & 49.24 & 1768.44 & $0.47^{\mathrm{b}}$ & 1.43 & 0.90 & 6.22 \\
\hline Rhamdia branneri Hanseman, 1911 & 19 & piscivorous & 36.50 & $49.77 *$ & 1822.22 & 0.24 & 1.17 & 0.53 & 5.88 \\
\hline Rhamdia quelen (Quoy \& Gaimard, 1824) & 125 & piscivorous & 39.40 & $39.17 *$ & 7746.39 & 0.01 & 1.01 & 0.35 & 4.25 \\
\hline Rhaphiodon vulpinus Spix \& Agassiz, 1829 & 3 & piscivorous & 44.00 & 46.20 & 508.19 & $0.39^{\mathrm{b}}$ & 1.76 & 0.70 & 8.55 \\
\hline Salminus brasiliensis (Cuvier, 1816) & 7 & piscivorous & 52.00 & 54.60 & 2077.30 & $0.20^{\mathrm{b}}$ & 1.43 & 0.40 & 6.02 \\
\hline Serrasalmus maculatus Kner, 1858 & 69 & piscivorous & 22.00 & 23.10 & 325.81 & $0.45^{\mathrm{b}}$ & 2.35 & 1.50 & 10.48 \\
\hline Serrasalmus marginatus Valenciennes, 1837 & 11 & piscivorous & 23.70 & 24.90 & 294.22 & $0.60^{\mathrm{b}}$ & 3.33 & 1.30 & 12.90 \\
\hline Steindachneridion scriptum (Miranda-Ribeiro, 1918) & 3 & piscivorous & 38.00 & $51.50 *$ & 1847.27 & $0.25^{\mathrm{a}}$ & 0.70 & 0.54 & 5.36 \\
\hline Steindachneridion melanodermatum Garavello, 2005 & 1 & piscivorous & 49.00 & $43.60 *$ & 1613.64 & $0.25^{\mathrm{a}}$ & 0.70 & 0.57 & 6.19 \\
\hline Tatia neivai (Ihering, 1930) & 20 & piscivorous & 9.50 & 10.00 & 11.78 & 0.60 & 0.55 & 1.51 & 14.63 \\
\hline Tatia jaracatia Pavanelli \& Bifi, 2009 & 5 & piscivorous & 6.60 & 6.90 & 6.00 & $0.50^{\mathrm{a}}$ & 0.80 & 1.49 & 17.60 \\
\hline
\end{tabular}

Rhamdia quelen is a catfish species, prefers calmer and deeper water along banks and vegetation with a sand or mud bottom (Silva et al., 2011). Despite the large distribution of this species, from northern Mexico to southern Argentina (Graça \& Pavanelli, 2007), studies on the biological aspects of this species have been primarily conducted on captive adult fish for farming purposes (Silva et al., 2011). Thus, comparative studies on the growth parameters for $R$. quelen were not found in field. On the other hand, Oreochromis niloticus showed rapid growth, reaching adult size in small interval of time, exhibit relatively short reproductive cycles, tolerate a variety of environmental conditions, have high capacity for hybridization and high resistance to diseases and infection (Coward \& Bromage, 2000). These biological features might have favored the rapid growth of $O$. niloticus populations in the reservoirs studied.

Natural mortality (M) in fish is difficult to estimate because its values are strongly correlated with growth parameters, as reported by Pauly (1998a). Fish with fast initial growth (higher $\mathrm{k}$ values) present higher $\mathrm{M}$ values, whereas fish with higher asymptotic lengths present lower $M$ values. The von Bertalanffy's growth constant and natural mortality were stronger correlated in this study, consequence of the fact that $\mathrm{M}$ was calculated using the Pauly's equation and then totally dependent on $\mathrm{k}$. 


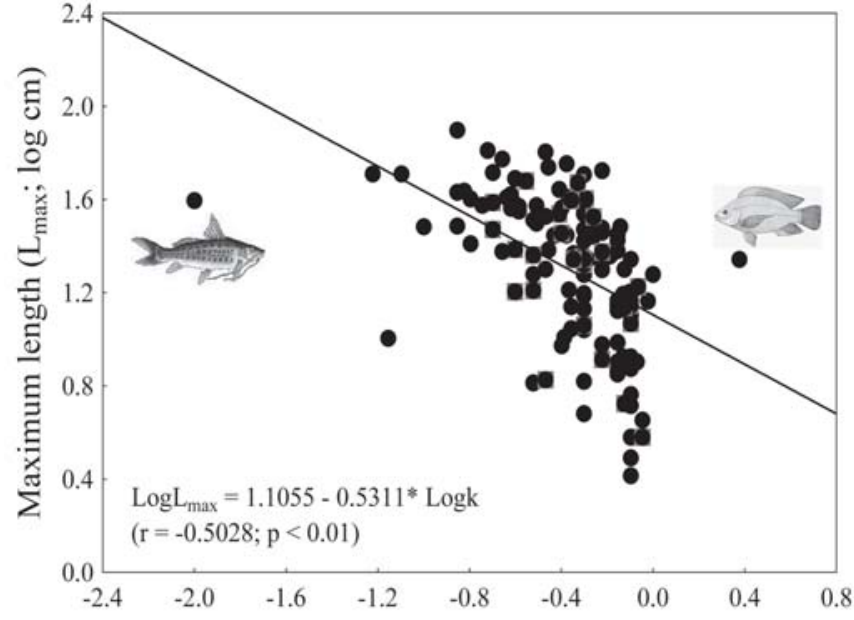

a von Bertalanffy growth constant $\left(\mathrm{k} ; \log \mathrm{yr}^{-1}\right)$
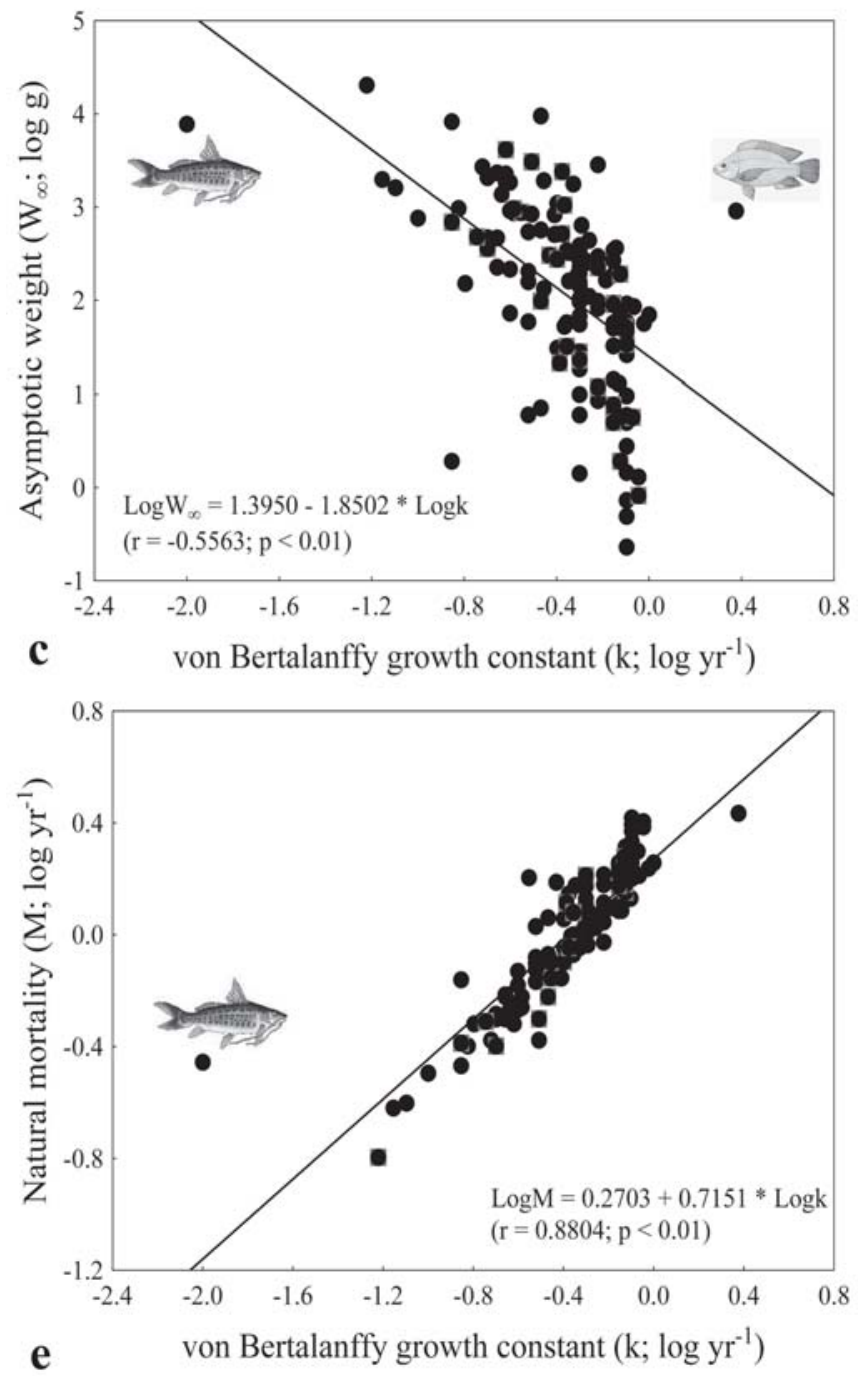
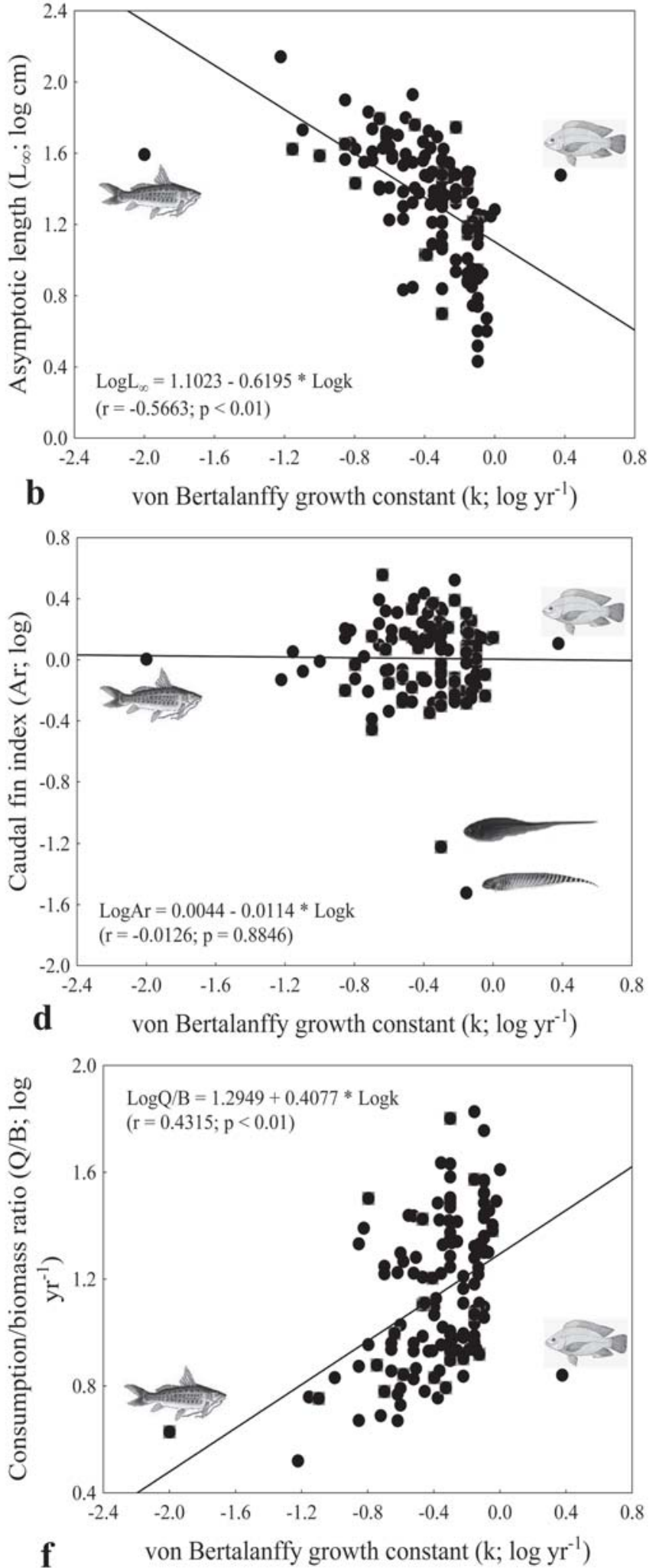

Fig. 2. Relationships between von Bertalanffy's constant ( $\mathrm{k}$; Growth rate) and maximum length ( $\left.\mathrm{L}_{\max } ; \mathbf{a}\right)$, asymptotic length $\left(\mathrm{L}_{o} ; \mathbf{b}\right)$, asymptotic weight $\left(\mathrm{W}_{o \rho} ; \mathbf{c}\right)$, caudal fin index $(\mathrm{Ar} ; \mathbf{d})$, natural mortality $(\mathrm{M} ; \mathbf{e})$ and consumption/biomass ratio $(\mathrm{Q} / \mathrm{B} ; \mathbf{f})$ for 135 fish species in reservoirs from the State of Paraná, southern Brazil, caught in July and November 2001. 

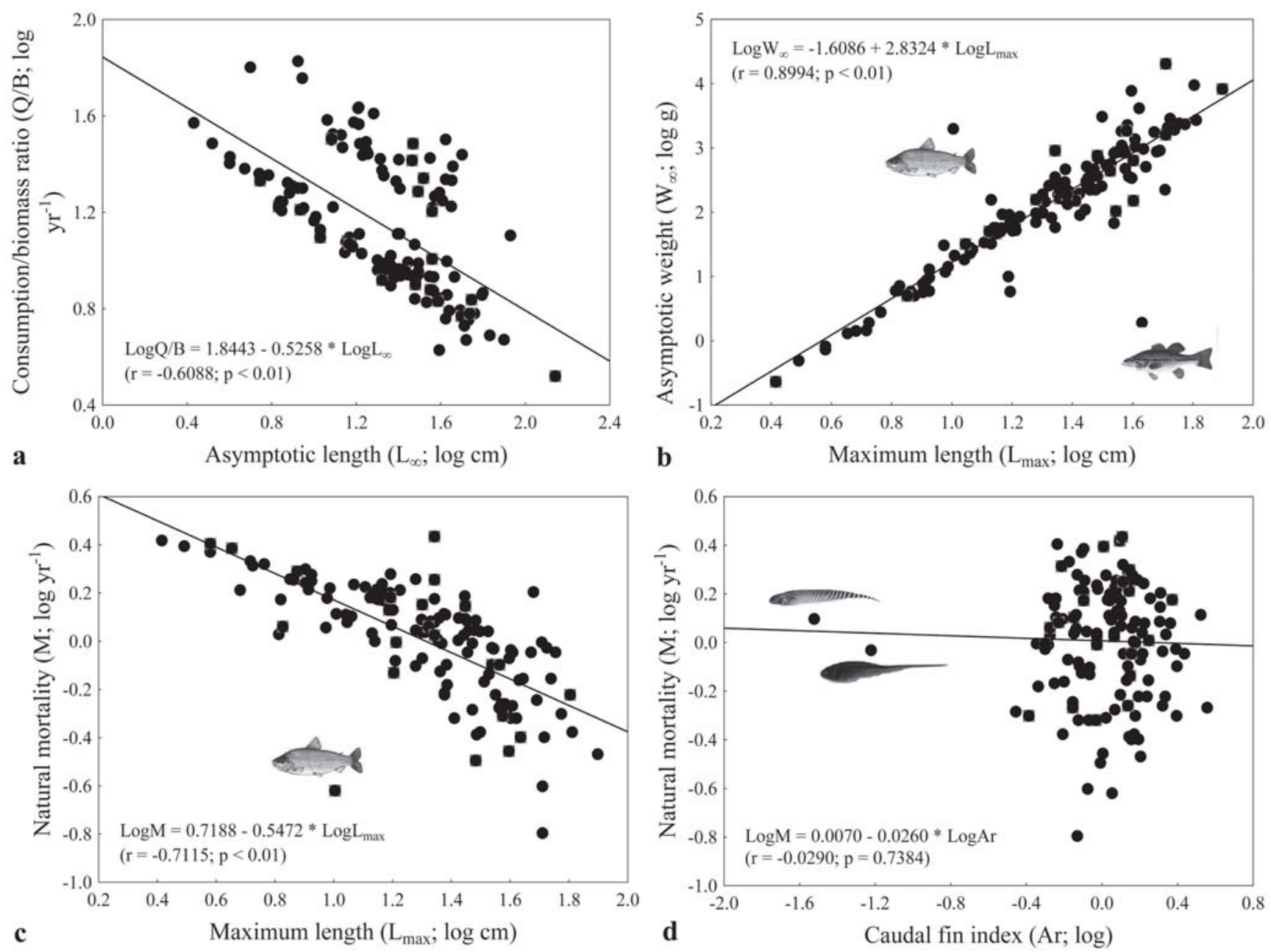

Fig. 3. Relationships between some biological parameters of 135 fish species in the State of Paraná, southern Brazil, caught in July and November 2001. Asymptotic length $\left(\mathrm{L}_{o}\right)$ and consumption/biomass ratio $\left(\mathrm{Q} / \mathrm{B}\right.$; a); maximum length $\left(\mathrm{L}_{\max }\right)$ and asymptotic weight $\left(\mathrm{W}_{o o} ; \mathbf{b}\right)$; maximum length $\left(\mathrm{L}_{\max }\right)$ and natural mortality $(\mathrm{M} ; \mathbf{c})$; and caudal fin index (Ar) and natural mortality (M; d).

The knifefishes species, as G. sylvius, E. trilineata, and Rhamphichthys hahni, showed the body shape highly specialized. The caudal portion of the body is elongate and it has a long anal fin, there are no dorsal, adipose, or pelvic fins, while the caudal fin is either small or absent (Albert, 2001). Thus, the caudal fin index for these species does not follow the specified pattern for other species. In addition, these species can present large maximum length, with such a high natural mortality, because on the shape of the body.

Consumption/Biomass ratio is defined as the number of times a population consumes its own weight within a year (Pauly, 1986, 1998a, 1998b; García \& Duarte, 2002). García \& Duarte (2002) studied Q/B ratio and estimates of Q/B-predictor parameters for Caribbean fish, and Angelini \& Agostinho (2005a) studied parameter estimates for fish from the upper Paraná River Floodplain and from Itaipu Reservoir (Brazil), and they found that $\mathrm{Q} / \mathrm{B}$ varies inversely with $\mathrm{L}_{o o}$ and trophic levels (species from low trophic levels showed high $\mathrm{Q} / \mathrm{B}$ ratio, due to their fast life cycles). Small-sized species, which are normally r-strategists (sensu Winemiller, 1989), showed low asymptotic lengths, short life cycles, high growth rates, early first maturation, high natural mortality, and high fecundity (Vazzoler, 1996). These species usually form the base of food webs, as they are concentrated in the lower trophic levels. Thus, patterns found in this study are similar to those observed previously.

The correlations found between the parameters studied here corroborate the patterns described by Pauly (1998a) and Angelini \& Agostinho (2005a), which indicated that $\mathrm{k}$ varies inversely with $\mathrm{L}_{o o}$ and $\mathrm{W}_{o}$, as well as $\mathrm{Q} / \mathrm{B}$ ratio varies inversely with $\mathrm{L}_{o}$ and natural mortality has a high correlation with $\mathrm{L}_{\max }$, for tropical fishes and also for fishes from other latitudes and ecosystems (Pauly, 1998b). Furthermore, we emphasize that, as suggested by Pauly (1998a), those correlations allow summarizing and comparing demographic and biological data of fish species from different biogeographic regions. 
The estimates presented here should be useful for building Ecopath models. Ecological modeling is under development in tropical regions, such as Brazil (Angelini \& Petrere, 1996; Wolff et al., 2000; Angelini \& Agostinho, 2005a, 2005b; Angelini et al., 2006; Angelini \& Gomes, 2008). Pauly (1998b) pointed out to the need for an adaptation or more precisely a "tropicalization" of the parameters from Beverton and Holt models. Moreover, García \& Duarte (2002) suggested that it would be interesting to explore the consistency of published consumption/biomass ratio values for the same species in similar habitats. Those authors compared estimated Q/B values with three empirical models described by Palomares \& Pauly $(1989,1998)$ and by Pauly et al., (1990), and they concluded that parameters vary for a given fish species; this is a clear sign that empirical models must be used with caution. Thus, empirical models obtained with data from temperate areas should be analyzed with attention. Furthermore, we emphasize that empirical models described by Palomares \& Pauly $(1989,1998)$ and by Pauly et al., (1990) should be parameterized with data from tropical areas.

\section{Acknowledgements}

We thank the Nupélia/UEM, PRONEX (Programa Nacional de Excelência) and Conselho Nacional de Desenvolvimento Científico e Tecnológico (CNPq) for the financial support and the grant for EAG. AAA and LCG are also granted by CNPq (Bolsa Produtividade). We also thank to Weferson Júnio da Graça by the review of list of species.

\section{Literature Cited}

Abelha, M. C. F. \& E. Goulart. 2004. Oportunismo trófico de Geophagus brasiliensis (Quoy \& Gaimard, 1824) (Osteichthyes, Cichlidae) no reservatório de Capivari, Estado do Paraná, Brasil. Acta Scientiarum. Biological Sciences, 26: 37-45.

Abelha, M. C. F., E. Goulart, E. A. L. Kashiwaqui \& M. R. Silva. 2006. Astyanax paranae Eigenmann, 1914 (Characiformes: Characidae) in the Alagados Reservoir, Paraná, Brazil: diet composition and variation. Neotropical Ichthyology, 4: 349-356.

Abelha, M. C. F., E. Goulart \& D. Peretti. 2005. Estrutura trófica e variação sazonal do espectro alimentar da assembléia de peixes do reservatório de Capivari, Paraná, Brasil. Pp. 197-209. In: Rodrigues, L., S. M. Thomaz, A. A. Agostinho \& L. C. Gomes (Eds.). Biocenoses em reservatórios: padrões espaciais e temporais. São Carlos, RiMa, 321p.

Abujanra, F., M. R. Russo \& N. S. Hahn. 1999. Variações espaçotemporais na alimentação de Pimelodus ortmanni (Siluriformes, Pimelodidae) no reservatório de segredo e áreas adjacentes (PR). Revista UNIMAR, 21: 283-289.

Agostinho, A. A., N. S. Hahn, L. C. Gomes \& L. M. Bini. 1997. Estrutura trófica. Pp. 229-248. In: Vazzoler, A. E. A. M., A. A. Agostinho \& N. S. Hahn (Eds.). A planície de inundação do alto rio Paraná: aspectos físicos, biológicos e socioeconômicos. Maringá, EDUEM, 460p.

Agostinho, A. A., L. C. Gomes \& F. M. Pelicice. 2007. Ecologia e manejo de recursos pesqueiros em reservatórios do Brasil. Maringá, Eduem, 507p.
Albert, J. S. 2001. Species diversity and phylogenetic systematic of American knifefishes (Gymnotiformes, Teleostei). Ann Arbor, Miscellaneous Publications, Museum of Zoology, University of Michigan, 126p.

Allen, K. R. 1971. Relation between production and biomass. Journal of the Fisheries Research Board of Canada, 28: 1573-1581.

Almeida, V. L. L., N. S. Hahn \& A. E. A. M. Vazzoler. 1997. Feeding patterns in five predatory fishes of the high Paraná River floodplain (Brazil). Ecology of Freshwater Fish, 6: 123-133.

Andrian, I. F. \& G. Barbieri. 1996. Espectro alimentar e variações sazonal e espacial na composição da dieta de Parauchenipterus galeatus Linnaeus, 1766, (Siluriformes, Auchenipteridae) na região do Reservatório de Itaipu (PR). Revista Brasileira de Biologia, 56: 409-422.

Andrian, I. F., C. C. R. C. Doria, G. Torrente \& C. M. L. Ferreti. 1994. Espectro alimentar e similaridade na composição da dieta de 4 espécies de Leporinus (Characiformes, Anostomidae) do rio Paraná, Brasil. Revista UNIMAR, 16: 97-106.

Angelini, R. \& A. A. Agostinho. 2005a. Parameter estimates for fishes of the Upper Paraná River Floodplain and Itaipu Reservoir (Brazil). NAGA, 28: 53-57.

Angelini, R. \& A. A. Agostinho. 2005b. Food web model of the Upper Paraná River Floodplain: description and aggregation effects. Ecological Modelling, 181: 109-121.

Angelini, R. \& M. Petrere Junior. 1996. The ecosystem of Broa Reservoir, São Paulo State, Brazil, as described using ECOPATH. NAGA, 19: 36-41.

Angelini, R. \& L. C. Gomes. 2008. O artesão dos ecossistemas: construindo modelos com dados. Maringá, EDUEM, 173p.

Angelini, R., A. A. Agostinho \& L. C. Gomes. 2006. Modeling energy flow in a large neotropical reservoir: a tool to evaluate fishing and stability. Neotropical Ichthyology, 4: 253-260.

Bennemann, S. T., A. M. Gealh, M. L. Orsi \& L. M. Souza. 2005. Ocorrência e ecologia trófica de quatro espécies de Astyanax (Characidae) em diferentes rios da bacia do rio Tibagi, Paraná, Brasil. Iheringia. Série Zoologia, 95: 247-254.

Bennemann, S. T., L. G. Capra, W. Galves \& O. A. Shibatta. 2006. Dinâmica trófica de Plagioscion squamosissimus (Perciformes, Sciaenidae) em trechos de influência da represa Capivara (rios Paranapanema e Tibagi). Iheringia. Série Zoologia, 96: 115-119.

Beverton, R. 1998. Fish, fact and fantasy: a long view. Reviews in Fish Biology and Fisheries, 8: 229-249.

Beverton, R. J. H. \& S. J. Holt. 1959. A review of the lifespans and mortality rates of fish in nature, and their relation to growth and other physiological characteristics. Pp. 142-180. In: Wohstenholme, G. E. \& M. O'Conner (Eds.). The lifespan of animals. Volume V, London, Churcill, 344p.

Binohlan, C. \& D. Pauly. 2000. The Popgrowth table. Pp. 138-145. In: Froese, R. \& D. Pauly (Eds.). Fishbase 2000: concepts, design and data sources. Los Baños, Laguna, Iclarm, 240p.

Cantanhêde, G., N. S. Hahn, E. A. Gubiani \& R. Fugi. 2008. Invasive molluses in the diet of Pterodoras granulosus (Valenciennes, 1821) (Pisces, Doradidae) in the Upper Paraná River floodplain, Brazil. Ecology of Freshwater Fish, 17: 47-53.

Cassemiro, F. A. S., N. S. Hahn \& R. Fugi. 2002. Avaliação da dieta de Astyanax altiparanae Garutti \& Britski, 2000 (Osteichthyes, Tetragonopterinae) antes e após a formação do reservatório de Salto Caxias, Estado do Paraná, Brasil. Acta Scientiarum. Biological Sciences, 24: 419-425.

Cassemiro, F. A. S., N. S. Hahn \& T. F. L. V. B. Rangel. 2003. Diet and trophic ecomorphology of the silverside, Odontesthes 
bonariensis, of the Salto Caxias reservoir, rio Iguaçu, Paraná, Brazil. Neotropical Ichthyology, 1: 127-131.

Cassemiro, F. A. S., N. S. Hahn \& R. L. Delariva. 2005. Estrutura trófica da ictiofauna, ao longo do gradiente longitudinal do reservatório de Salto Caxias (rio Iguaçu, Paraná, Brasil), no terceiro ano após o represamento. Acta Scientiarum. Biological Sciences, 27: 63-71.

Christensen, V. \& D. Pauly. 1993. Trophic models of aquatic ecosystems. International Center for Living Aquatic Resources Management, International Council for the Exploration of the Sea, Danish International Development Agency, Makati, Metro Manila, Philippines, Copenhagen K., Denmark, 390p.

Comitê Brasileiro de Barragens. 2007: Available at: http:// www.cbdb.org.br/site/listabarragens.asp (last accessed on 14 March 2007).

Companhia Paranaense de Energia Elétrica. 2007: Available at: http:/ /www.copel.com/pagcopel.nsf/secaos/ger_usinas (last accessed on 14 March 2007).

Coward, K. \& N. R. Bromage. 2000. Reproductive physiology of female tilapia broodstock. Reviews in Fish Biology and Fisheries, 10: $1-25$.

Dias, R. M., D. Bailly, R. R. Antônio, H. I. Suzuki \& A. A. Agostinho. 2005. Colonization of the Corumbá Reservoir (Corumbá River, Paraná River Basin, Goiás State, Brazil) by the "lambari" Astyanax altiparanae (Tetragonopterinae; Characidae). Brazilian Archives of Biology and Technology, 48: 467-476.

Ferreti, C. M. L., I. F. Andrian \& G. Torrente. 1996. Dieta de duas espécies de Schizodon (Characiformes, Anostomidae), na planície de inundação do alto rio Paraná e sua relação com aspectos morfológicos. Boletim do Instituto de Pesca, 23: 171-186.

Froese, R. \& C. Binohlan. 2000. Empirical relationships to estimate asymptotic length, length at first maturity, and length at maximum yield per recruit in fishes, with a simple method to evaluate length frequency data. Journal of Fish Biology, 56: 758-773.

Froese, R. \& C. Binohlan. 2003. Simple methods to obtain preliminary growth estimates for fishes. Journal of Applied Ichthyology, 19: 376-379.

Froese, R. \& D. Pauly. 2000. Fishbase 2000: Concepts, design and data sources. ICLARM, Los Baños, Laguna, Philippines, 344p.

Froese, R. \& D. Pauly. 2010. FishBase. World Wide Web electronic publication. www.fishbase.org, version (05/2010).

Fugi, R. \& N. S. Hahn. 1991. Espectro alimentar e relações morfológicas com o aparelho digestivo de 3 espécies de peixes comedoras de fundo do Rio Paraná. Revista Brasileira de Biologia, 51: 873-879.

Fugi, R., A. A. Agostinho \& N. S. Hahn. 2001. Trophic morphology of five benthic-feeding fish species of a tropical floodplain. Revista Brasileira de Biologia, 61: 27-33.

Fugi, R., N. S. Hahn, V. Loureiro-Crippa \& G. C. Novakowski. 2005. Estrutura trófica da ictiofauna em reservatórios. Pp. 185195. In: Rodrigues, L., S. M. Thomaz, A. A. Agostinho \& L. C. Gomes (Eds.). Biocenoses em reservatórios: padrões espaciais e temporais. São Carlos, RiMa, 321p.

García, C. B. \& L. O. Duarte. 2002. Consumption to biomass (Q/B) ratio and estimates of $\mathrm{Q} / \mathrm{B}$-predictor parameters for Caribbean fishes. NAGA, 25: 19-31.

Gayanilo, F. C. Jr. \& D. Pauly. 1997. FAO-ICLARM stock assessment tools (FiSAT) - Reference manual. FAO Computerized Information Series (Fisheries). No. 8. FAO, Rome, 262p.

Gealh, A. M. \& N. S. Hahn. 1998. Alimentação de Oligosarcus longirostris Menezes \& Gèry (Osteichthyes,
Acestrorhynchinae) do reservatório de Salto Segredo, Paraná, Brasil. Revista Brasileira de Zoologia, 15: 985-993.

Graça, W. J. \& C. S. Pavanelli. 2007. Peixes da planície de inundação do alto rio Paraná e áreas adjacentes. Maringá, EDUEM, 241p.

Gulland, J. A. 1977. Fish population dynamics. New York, John Wiley \& Sons, 372p.

Hahn, N. S., R. Fugi \& I. F. Andrian. 1991. Espectro e atividade alimentares do armadinho, Trachydoras paraguayensis (Doradidae; Siluriformes) em distintos ambientes do Rio Paraná. Revista UNIMAR, 13: 177-194.

Hahn, N. S., A. Monfredinho, R. Fugi \& A. A. Agostinho. 1992. Aspectos da alimentação do armado, Pterodoras granulosus (Ostariophysi, Doradidae) em distintos ambientes do alto rio Paraná. Revista UNIMAR, 14: 163-176.

Hahn, N. S., R. Fugi, V. L. L. Almeida, M. R. Russo \& V. E. Loureiro. 1997a. Dieta e atividade alimentar de peixes do reservatório de Segredo. Pp. 143-162. In: Agostinho, A. A. \& L. C. Gomes (Eds.). Reservatório de Segredo: bases ecológicas para o manejo. Maringá, EDUEM, 387p.

Hahn, N. S., I. F. Andrian, R. Fugi \& V. L. L. Almeida. 1997b. Ecologia trófica. Pp. 209-228. In: Vazzoler, A. E. A. M., A. A. Agostinho \& N. S. Hahn (Eds.). A planície de inundação do alto rio Paraná: aspectos físicos, biológicos e socioeconômicos. Maringá, EDUEM, 460p.

Hahn, N. S., A. A. Agostinho, L. C. Gomes \& L. M. Bini. 1998. Estrutura trófica da ictiofauna do reservatório de Itaipu (PR Brasil) nos primeiros anos de sua formação. Interciencia, 23: 299-305.

Hahn, N. S., V. E. Loureiro \& R. L. Delariva. 1999. Atividade alimentar da curvina Plagioscion squamosissimus (Heckel, 1840) (Perciformes, Sciaenidae) no rio Paraná. Acta Scientiarum. Biological Sciences, 21: 309-314.

Holt, S. J. 1998. Fifty years on. Reviews in Fish Biology and Fisheries, 8: 357-366.

Ingenito, L. F. S., L. F. Duboc \& V. Abilhoa. 2004. Contribuição ao conhecimento da ictiofauna da bacia do alto rio Iguaçu, Paraná, Brasil. Arquivos de Ciências Veterinárias e Zoologia da UNIPAR, 7: 23-36.

Ivlev, V. S. 1945. The biological productivity of waters. Translated by Ricker, W. E. 1966. Journal of the Fisheries Research Board of Canada, 23: 1727-1759.

Jarre, A., M. L. Palomares, M. L. Soriano, V. C. Sambilay \& D. Pauly. 1991. Some new analytical and comparative methods for estimating the food consumption of fish. International Council for the Exploration of the Sea Marine Science Symposium, 193: 99-108.

Jensen, A. L. 1997. Origin of the relation between $\mathrm{K}$ and $\mathrm{L}_{\mathrm{inf}}$ and synthesis of relations among life history parameters. Canadian Journal of Fisheries and Aquatic Sciences, 54: 987-989.

Júlio Jr, H. F., S. M. Thomaz, A. A. Agostinho \& J. D. Latini. 2005. Distribuição e caracterização dos reservatórios. Pp. 1-16. In: Rodrigues, L., S. M. Thomaz, A. A. Agostinho \& L. C. Gomes (Eds.). Biocenoses em reservatórios: padrões espaciais e temporais. São Carlos, RiMa, 321p.

Lolis, A. A. \& I. F. Andrian. 1996. Alimentação de Pimelodus maculatus Lacépede, 1803 (Siluriformes, Pimelodidae) na planície de inundação do alto rio Paraná, Brasil. Boletim do Instituto de Pesca, 23: 187-202.

Loureiro, V. E. \& N. S. Hahn. 1996. Dieta e atividade alimentar da traíra Hoplias malabaricus (Block, 1794) (Osteichthyes, Erythrinidae) nos primeiros anos de formação do reservatório de Segredo. Acta Limnologica Brasiliensia, 8: 195-205. 
Loureiro-Crippa, V. E. \& N. S. Hahn. 2006. Use of food resources by the fish fauna of a small reservoir (rio Jordão, Brazil) before and shortly after its filling. Neotropical Ichthyology, 4: 357-362.

Menezes, N. A., S. H. Weitzman, O. T. Oyakawa, F. C. T. Lima, R. M. C. Castro \& M. J. Weitzman. 2007. Peixes de água doce da Mata Atlântica: lista preliminar das espécies e comentários sobre conservação de peixes de água doce neotropicais. São Paulo, Museu de Zoologia, Universidade de São Paulo, 407p.

Nelson, J. S. 2006. Fishes of the World. New York, John Wiley \& Sons, $601 \mathrm{p}$.

Nikolsky, G. V. 1969. Theory of fish population dynamics. Edinburgh, Oliver \& Boyd, 323p.

Oricolli, M. C. G. \& S. T. Bennemann. 2006. Dieta de Bryconamericus iheringii (Ostariophysi:Characidae) em riachos da bacia do rio Tibagi. Acta Scientiarum. Biological Sciences, 28: 59-63.

Ortêncio Filho, H., N. S. Hahn, R. Fugi \& M. R. Russo. 2001. Aspectos da alimentação de Glanidium ribeiroi (Haseman, 1911) (Teleostei, Auchenipteridae), espécie endêmica do rio Iguaçu, PR. Acta Limnologica Brasiliensia, 13: 85-92.

Oyakawa, O. T., A. Akama, K. C. Mautari \& J. C. Nolasco. 2006. Peixes de riachos da mata Atlântica nas unidades de conservação do vale do rio Ribeira de Iguape no Estado de São Paulo. São Paulo, Neotrópica, 201p.

Palomares, M. L. D. \& D. Pauly. 1989. A multiple regression model for predicting the food consumption of marine fish populations. Australian Journal of Marine \& Freshwater Research, 40: 259-273.

Palomares, M. L. D. \& D. Pauly. 1998. Predicting food consumption of fish populations as functions of mortality, food type, morphometrics, temperature and salinity. Marine and Freshwater Research, 49: 447-453.

Pauly, D. 1980. On the interrelationships between natural mortality, growth parameters and mean environmental temperature in 175 fish stocks. Journal du Conseil International pour I'Exploration de la Mer, 39: 175-192.

Pauly, D. 1986. A Simple Method for Estimating the food consumption of fish populations from growth data and food conversion experiments. Fishery Bulletin (U.S.), 84: 827-840.

Pauly, D. 1989. Food consumption by tropical and temperate fish populations: some generalizations. Journal of Fish Biology, 35: $11-20$

Pauly, D. 1998a. Tropical fishes: patterns and propensities. Journal of Fish Biology, 53: 1-17.

Pauly, D. 1998b. Beyond our original horizons: the tropicalization of Beverton and Holt. Reviews in Fish Biology and Fisheries, 8: 307-334

Pauly, D., V. Christensen \& V. C. Sambilay. 1990. Some features of fish food consumption estimates used by ecosystem modelers. London, ICES Council Meeting, 9p.
Pauly, D., V. Christensen \& C. Walters. 2000. Ecopath, Ecosim, and Ecospace as tools for evaluating ecosystem impact of fisheries. ICES Journal of Marine Science, 57: 697-706.

Perez Lizama, M. de Los A. \& A. E. A. M. Vazzoler. 1993. Crescimento em peixes do Brasil: uma síntese comentada. Revista UNIMAR, 15(Suplemento): 141-173.

Ricker, W. E. 1975. Computation and interpretation of biological statistics of fish populations. Bulletin of the Fisheries Research Board of Canada, 191: 1-382.

Russo, M. R., N. S. Hahn \& C. S. Pavanelli. 2004. Resource partitioning between two species of Bryconamericus Eigenmann, 1907 from the Iguaçu river basin, Brazil. Acta Scientiarum. Biological Sciences, 26: 431-436.

Severi, W. \& A. A. M. Cordeiro. 1994. Catálogo de peixes da bacia do rio Iguaçu. Curitiba, IAP/GTZ, 128p.

Silva, E. B., P. H. S. Picapedra, P. V. Sanches. R. E. O. Rezende, A. M. Gavião, M. M. Mendonça, E. D. V. Gonçalves \& R. B. Conte. 2011. Larvae occurrences of Rhamdia quelen (Quoy \& Gaimard, 1824) (Siluriformes: Heptapteridae) in an area under dam influence in the upper Paraná River region, Brazil. Neotropical Ichthyology, 9: 419-426.

Souza-Stevaux, M. C., R. R. B. Negrelle \& V. Citadini-Zanette. 1994. Seed dispersal by the fish Pterodoras granulosus in the Paraná River Basin, Brazil. Journal of Tropical Ecology, 10: 621-626.

Sparre, P., E. Ursin \& S. C. Venema. 1989. Introduction to tropical fish stock assessment. Part 1. Manual. Fisheries technical paper, Rome, FAO, 337p.

Stergiou, K. I. \& P. K. Karachle. 2006. A review of various biological parameters for fish from the Greek Seas. Journal of Biological Research, 6: 199-211.

Stergiou, K. I. \& V. S. Karpouzi. 2002. Feeding habitats and trophic levels of Mediterranean fishes. Reviews in Fish Biology and Fisheris, 11: 217-254

Thornton, K. W., B. L. Kimmel \& F. E. Payne. 1990. Reservoir limnology: ecological perspectives. New York, John Wiley \& Sons, 246p.

Vazzoler, A. E. A. M. 1996. Biologia da reprodução de peixes teleósteos: Teoria e prática. Maringá, EDUEM, 169p.

Winemiller, K. O. 1989. Patterns of variation in life history among South American fishes in seasonal environments. Oecologia, 81: 225-241.

Wolff, M., V. Koch \& V. Isaac. 2000. A trophic model of Caeté Mangrove Estuary (North Brazil) with considerations for the sustainable use of its resources. Estuarine, Coastal and Shelf Science, 50: 789-803.

Submitted April 1, 2011

Accepted September 26, 2011

Published March 30, 2012 